\title{
Chemoenzymatic and Template-Directed Synthesis of Bioactive Macrocyclic Peptides
}

\author{
Jan Grünewald and Mohamed A. Marahiel* \\ Fachbereich Chemie/Biochemie, Philipps-Universität Marburg, Hans-Meerwein-Strasse, D-35032 Marburg, Germany
}

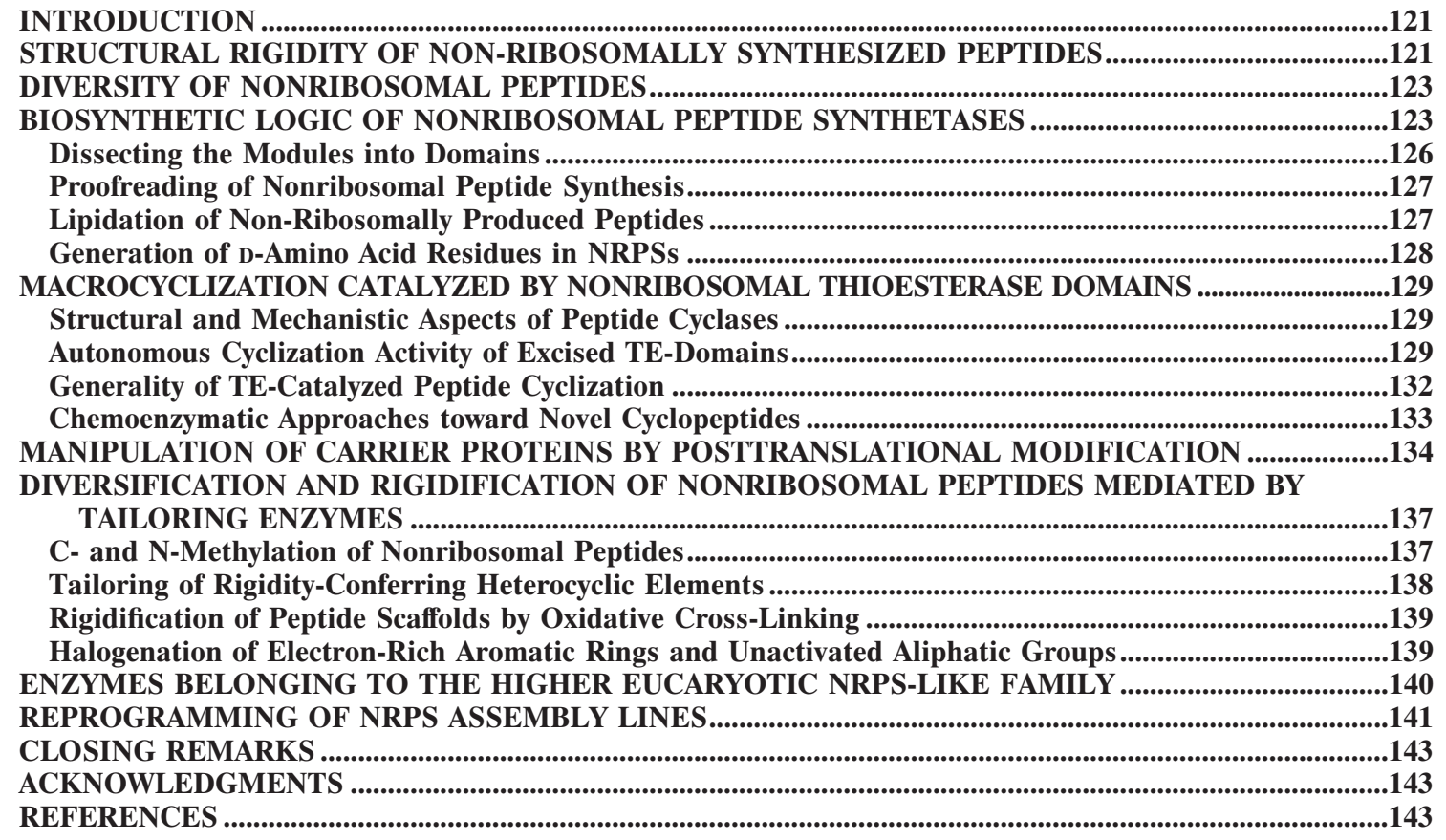

\section{INTRODUCTION}

Natural products that are produced by microorganisms have for decades attracted considerable attention for modern therapy. The bioactivity of these structurally complex substances extends from antibiotic to immunosuppressive and from cytostatic to antitumor (106). Not only have these secondary metabolites been optimized for their dedicated function over millions of years of evolution, they also represent promising scaffolds for the development of novel drugs with improved or altered activities. Optimization can be achieved by the introduction of artificial modifications, which yields semisynthetic derivatives of existing structures, although total synthesis of complete natural-product-based compounds is also envisioned $(138,144)$.

Peptidic products represent a large subclass of highly diverse natural products, many of which display therapeutically useful activity. They can be classified into different groups according to their synthesis pathways. The lantibiotics, for example, are ribosomally synthesized antimicrobial agents that are posttranslationally modified to their biologically active forms (18). Yet another class, a widespread class of therapeutically important

\footnotetext{
* Corresponding author. Mailing address: Fachbereich Chemie/Biochemie, Philipps-Universität Marburg, Hans-Meerwein-Strasse, D-35032 Marburg, Germany. Phone: 49-6421-2825722. Fax: 49-6421-2822191. E-mail: marahiel@chemie.uni-marburg.de.
}

peptides, are produced nonribosomally by large multienzyme complexes, the nonribosomal peptide synthetases (NRPS) (81, 111). In contrast to ribosomal peptide synthesis, non-ribosomally assembled peptides contain not only the common 20 amino acids (aa) but hundreds of different building blocks. Moreover, these secondary metabolite peptides contain unique structural features, such as D-amino acids, N-terminally attached fatty acid chains, $\mathrm{N}$ - and $\mathrm{C}$-methylated residues, $\mathrm{N}$ formylated residues, heterocyclic elements, and glycosylated amino acids, as well as phosphorylated residues (111). In recent research using both genetic and biochemical methods, experiments have revealed deep insights into the mechanism of nonribosomal peptide synthesis. In many cases, it was possible to alter existing non-ribosomally produced peptides by the combined action of chemical peptide synthesis and subsequent enzyme catalysis. This chemoenzymatic approach, along with a brief overview of the nonribosomal peptide synthesis machinery, will be discussed in more detail later in this review. Another focus of this article will be the labeling of NRPS-derived proteins by site-specific posttranslational modification.

\section{STRUCTURAL RIGIDITY OF NON-RIBOSOMALLY SYNTHESIZED PEPTIDES}

Selected structures of some non-ribosomally produced peptides are shown in Fig. 1. A common feature of these com- 


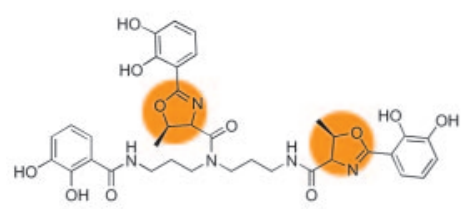

1: vibriobactin
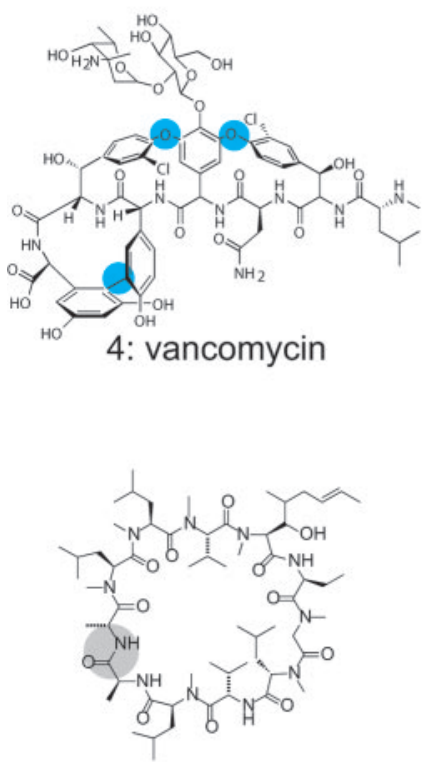

7: cyclosporin

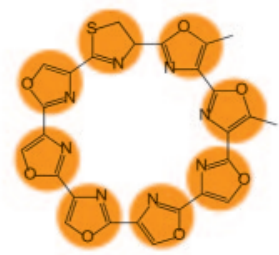

2: telomestatin

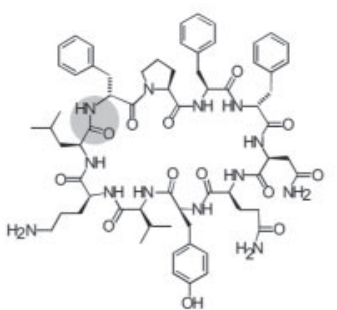

5: tyrocidine $A$

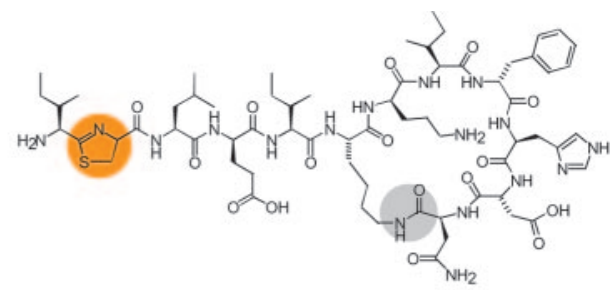

3: bacitracin

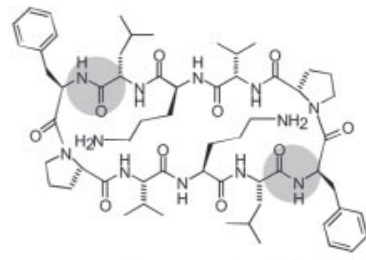

6: gramicidin S

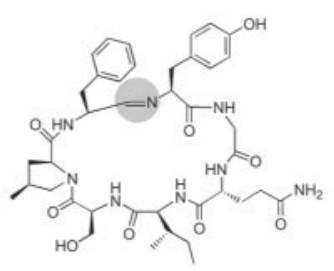

8: nostocyclopeptide
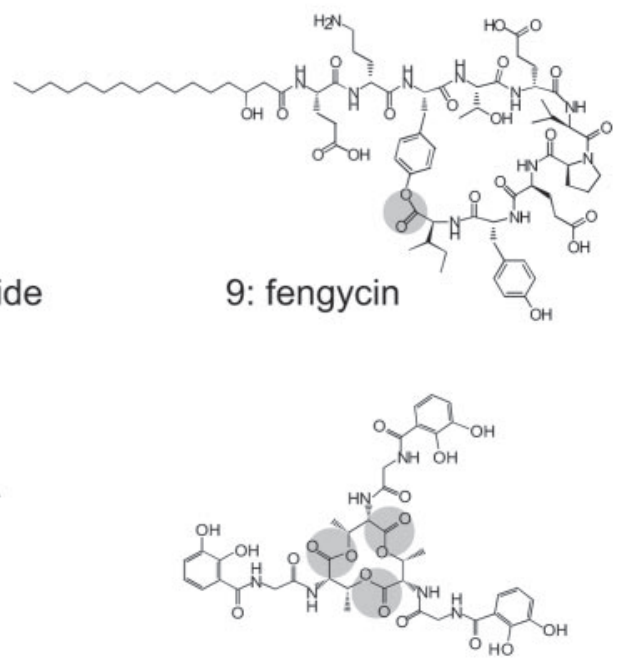

12: bacillibactin

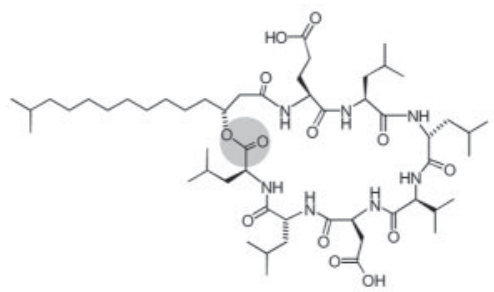

11: surfactin $A$

\section{heterocyclization}

\section{cross-linking}

FIG. 1. A selection of non-ribosomally synthesized peptides. Characteristic structural features that confer rigidity to the peptide backbone are highlighted.

pounds is their constrained structure, which ensures bioactivity by a precise orientation required for interaction with a dedicated molecular target (68). In some cases, these constraints are imposed by heterocyclization. For instance, the iron-chelating siderophore vibriobactin comprises two oxazoline rings, both of which originate from threonine residues (145). This oxazoline ring can be further oxidized to yield oxazole, as found in the potent telomerase inhibitor telomestatin (139). In addition to oxazoles, telomestatin also contains a thiazoline ring that is synthesized by the heterocyclization of cysteine. In the case of the antibiotic bacitracin, this heterocyclic element mediates a specific cation-dependent complexation of the phosphate group of the $\mathrm{C}_{55}$ lipid carrier, leading to depletion of this carrier and subsequent blocking of bacterial cell wall synthesis $(122,123)$. An additional strategy to modify and thus constrain the conformation of nonribosomal peptides is exemplified by the glycopeptide antibiotics of the vancomycin and teicoplanin class (57). These closely related compounds contain a homologous heptapeptide scaffold, whose backbone is constrained by extensive oxidative cross-linking. The joining of electron-rich aromatic rings by aryl ether linkages and direct C-C coupling convert these acyclic, floppy heptapeptides into rigid, cup-shaped structures. The constrained glycopeptides sequester the $N$-acyl-D-Ala-D-Ala termini of bacterial peptido- 
glycan strands with five hydrogen bonds and inhibit the transglycosylation and/or transpeptidation steps of bacterial peptidoglycan synthesis $(4,143)$.

Macrocyclization is another common constraint of non-ribosomally synthesized peptides whereby parts of the molecule distant in the linear peptide precursor are covalently linked to one another (68). Thus far, many biological strategies for the cyclization of nonribosomal cyclopeptides have been identified, giving rise to a high diversity in this class of compounds. For instance, the intramolecular capture by amines leads to peptidolactams, whereas cyclization via hydroxyl substituents leads to peptidolactones. The former strategy is observed for the peptide antibiotics tyrocidine $\mathrm{A}$, bacitracin, gramicidin $\mathrm{S}$ (110), and the immunosuppressive drug cyclosporine (77). In the case of tyrocidine A, amide bond formation occurs headto-tail between the $\mathrm{N}$-terminal amino group and the $\mathrm{C}$ terminus of the decapeptide. An unusual type of head-to-tail cyclization is observed for nostocyclopeptide (42), where the terminal ends of the peptide are linked via an imine bond. In contrast, the dodecapeptide bacitracin has a lariat structure, with the heptapeptide lactam ring arising from capture of the C-terminal carbonyl group by the $\varepsilon$-amino group of $\mathrm{Lys}_{6}$. Moreover, the macrolactam gramicidin $\mathrm{S}$ is composed of two identical pentapeptides bridged head-to-tail yielding a symmetric dilactam ring. For macrolactones, analogous cyclization strategies lead to branched-cyclic structures as seen for the antifungal lipopeptide fengycin, the antibiotic pristinamycin (63), and the biosurfactant surfactin A (110). The former depsipeptides are cyclized via the side chains of hydroxy amino acids such as tyrosine and threonine, whereas the latter compound is cyclized via a $\beta$-hydroxylated fatty acid moiety. Finally, the ironchelating siderophore bacillibactin is a cyclic trilactone that arises from cyclotrimerization of threonine (84).

\section{DIVERSITY OF NONRIBOSOMAL PEPTIDES}

The structural diversity of non-ribosomally produced peptides is best exemplified for the class of acidic lipopeptide antibiotics, including the calcium-dependent antibiotic (CDA) from Streptomyces coelicolor (51), daptomycin from Streptomyces roseosporus $(3,99)$ and A54145 from Streptomyces fradiae $(39,86)$, as well as friulimicins and amphomycins from Actinoplanes friuliensis (136). All of these lipopeptides originate from streptomycetes, which produce over two-thirds of naturally derived antibiotics (8). Each member of this class of lipopeptides can be subdivided into various individual compounds that differ in the structure of the $\mathrm{N}$ terminally attached fatty acid moiety and/or the peptide backbone (Fig. 2). For example, A54145 is a complex of eight lipopeptides which are acylated with an 8-methylnonanoyl, $n$-decanoyl, or 8-methyldecanoyl lipid side chain. These factors also contain four different cyclic peptide nuclei which differ in glutamate/3-methylglutamate (position 12) and/or valine/isoleucine (position 13) substitutions (39). The diversity of acidic lipopeptide antibiotics is further amplified by the occurrence of D-configured as well as nonproteinogenic amino acids, including D-4-hydroxyphenylglycine (D-HPG), D-3-phosphohydroxyasparagine, 3-methylglutamate (3mGlu), D-pipecolic acid, kynurenine (Kyn), and many more. Interestingly, all of the acidic lipopeptide antibiotics are comprised of a branched cyclic decapeptide lactone ring or lactam ring. The positions of the D-configured amino acids are strictly conserved in this macrocyclic scaffold. Moreover, two aspartic acid residues are found in equivalent ring positions of the macrolactone or macrolactam ring. Recently, a genomics-based approach revealed the existence of numerous uncharacterized lipopeptide biosynthetic gene clusters, indicating that many more antibiotics of this class have yet to be identified (88).

The therapeutic importance of the acidic lipopeptide antibiotics is best exemplified for daptomycin. This amphiphatic tridecapeptide is a member of the A21978C complex produced by $S$. roseosporus (Fig. 2). Although the major components, $\mathrm{A}_{21978 \mathrm{C}_{1}}$

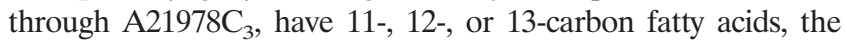
yield of daptomycin (10-carbon fatty acid) from fermentations is significantly increased by adding decanoic acid to the medium. Daptomycin (Cubicin; Cubist Pharmaceuticals), exhibits bactericidal activity against resistant pathogens for which there are very few therapeutic alternatives, such as vancomycin-resistant enterococci, methicillin-resistant Staphylococcus aureus, and penicillinresistant Streptococcus pneumoniae (126). At present, spontaneous acquisition of resistance to daptomycin is rare, which might be due to a unique mechanism of action (99).

Although the mechanism of action of daptomycin is not yet fully understood, it has been clearly established that calcium ions play an essential role in antimicrobial potency $(54,55)$. A nuclear magnetic resonance (NMR) study indicated that the stoichiometry of $\mathrm{Ca}^{2+}$ binding to daptomycin is one to one (2). Therefore, the total charge of the $\mathrm{Ca}^{2+}$-conjugated daptomycin $(-1)$ is lower than that of $\mathrm{Ca}^{2+}$-free daptomycin $(-3)$ at a neutral $\mathrm{pH}$. This would result in a more hydrophobic molecule due to charge neutralization, facilitating interaction of $\mathrm{Ca}^{2+}$ conjugated daptomycin with lipid bilayers. It has been proposed that, upon association with bacterial cytoplasmatic membranes, a major $\mathrm{Ca}^{2+}$-dependent conformational change promotes deeper insertion of daptomycin into the lipid bilayer (55). This is followed by large membrane perturbations, including lipid flip-flop and membrane leakage. Formation of any of these presumably disrupts the functional integrity of the membrane leading to cell death of gram-positive bacteria.

Although some of the key structural prerequisites for daptomycin's antibacterial activity have been identified, the exact nature of the molecular targets within the cytoplasmatic membrane has yet to be established. However, the aforementioned model of the mechanism of action provides an initial step toward understanding how this antibiotic gains access to and interacts with bacterial membranes. Since the other acidic lipopeptide antibiotics CDA, A54145, friulimicins, and amphomycins share key structural features with daptomycin; they might undergo similar interactions with calcium ions and bacterial membranes. Therefore, it is essential to further probe the structure-function relationship of all acidic lipopeptide antibiotics. Using this knowledge will enable the design of new and improved derivatives of this remarkable class of antibiotics. However, in order to engineer more potent variants, one has to understand the biosynthesis of these complex compounds. This will be the focus of the following section.

\section{BIOSYNTHETIC LOGIC OF NONRIBOSOMAL PEPTIDE SYNTHETASES}

Despite the structural diversity of the non-ribosomally produced acidic lipopeptide antibiotics, these secondary metabo- 


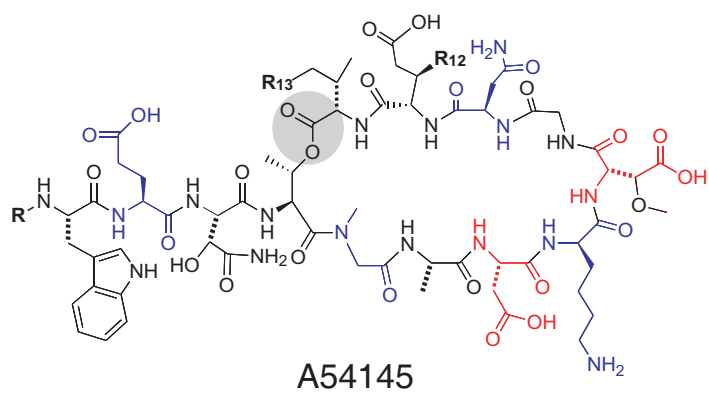

\begin{tabular}{|c|l|l|l|}
\hline factor & \multicolumn{1}{|c|}{$\mathbf{R}$} & $\mathbf{R} 12$ & $\mathbf{R} 13$ \\
\hline$A$ & 8-methylnonanoyl $\left(i \mathrm{C}_{10}\right)$ & $\mathrm{H}$ & $\mathrm{CH}_{3}$ \\
\hline $\mathrm{A}_{1}$ & n-decanoyl $\left(n \mathrm{C}_{10}\right)$ & $\mathrm{H}$ & $\mathrm{CH}_{3}$ \\
\hline $\mathrm{B}$ & n-decanoyl $\left(n \mathrm{C}_{10}\right)$ & $\mathrm{CH}_{3}$ & $\mathrm{CH}_{3}$ \\
\hline $\mathrm{B}_{1}$ & 8-methylnonanoyl $\left(i \mathrm{C}_{10}\right)$ & $\mathrm{CH}_{3}$ & $\mathrm{CH}_{3}$ \\
\hline $\mathrm{C}$ & 8-methyldecanoyl $\left(a \mathrm{C}_{11}\right)$ & $\mathrm{CH}_{3}$ & $\mathrm{H}$ \\
\hline$D$ & 8-methyldecanoyl $\left(a \mathrm{C}_{11}\right)$ & $\mathrm{H}$ & $\mathrm{CH}_{3}$ \\
\hline$E$ & 8-methyldecanoyl $\left(a \mathrm{C}_{11}\right)$ & $\mathrm{CH}_{3}$ & $\mathrm{CH}_{3}$ \\
\hline $\mathrm{F}$ & 8-methylnonanoyl $\left(i \mathrm{C}_{10}\right)$ & $\mathrm{H}$ & $\mathrm{H}$ \\
\hline
\end{tabular}

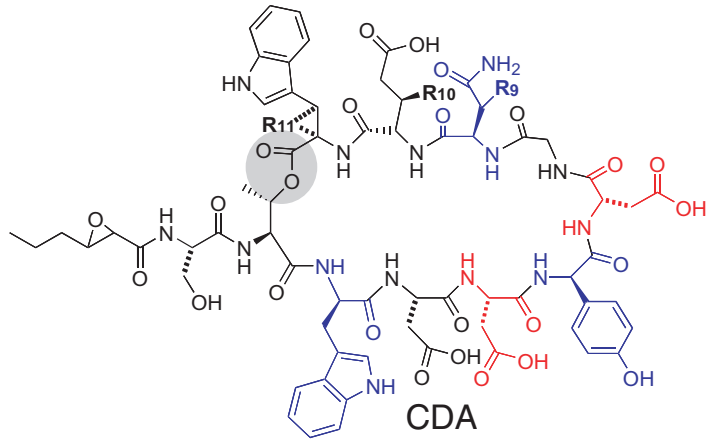

\begin{tabular}{|l|l|l|c|}
\hline factor & $\mathbf{R} 9$ & $\mathbf{R} 10$ & $\mathbf{R} 11$ \\
\hline $\mathrm{CDA1b}$ & $\mathrm{OPO}_{3} \mathrm{H}_{2}$ & $\mathrm{H}$ & $\mathrm{H}, \mathrm{H}$ \\
\hline $\mathrm{CDA} 2 \mathrm{a}$ & $\mathrm{OPO}_{3} \mathrm{H}_{2}$ & $\mathrm{CH}_{3}$ & $\pi$-bond \\
\hline $\mathrm{CDA} 2 \mathrm{~b}$ & $\mathrm{OPO}_{3} \mathrm{H}_{2}$ & $\mathrm{CH}_{3}$ & $\mathrm{H}, \mathrm{H}$ \\
\hline $\mathrm{CDA3a}$ & $\mathrm{OH}$ & $\mathrm{H}$ & $\pi$-bond \\
\hline $\mathrm{CDA3b}$ & $\mathrm{OH}$ & $\mathrm{H}$ & $\mathrm{H}, \mathrm{H}$ \\
\hline $\mathrm{CDA4a}$ & $\mathrm{OH}$ & $\mathrm{CH}_{3}$ & $\pi$-bond \\
\hline $\mathrm{CDA4b}$ & $\mathrm{OH}$ & $\mathrm{CH}_{3}$ & $\mathrm{H}, \mathrm{H}$ \\
\hline
\end{tabular}

FIG. 2. Diversity of acidic lipopeptide antibiotics. At least 27 compounds have been characterized so far. A54145 is produced by Streptomyces fradiae, daptomycin is produced by Streptomyces roseosporus, CDA is produced by Streptomyces coelicolor, and friulimicins and amphomycins are derived from Actinoplanes friuliensis. Conserved acidic residues are indicated in red, and D-configured/achiral residues at equivalent positions are highlighted in blue. The cyclization site is indicated by shading.

lites share a common mode of synthesis, the so-called "multiple carrier thio-template mechanism" $(75,76,81)$. According to this model, peptide synthesis is performed by nonribosomal peptide synthetases (NRPSs). Figure 3 shows the NRPS assembly lines for daptomycin, A54145 and CDA. Detailed analysis of the daptomycin gene cluster revealed that the daptomycin biosynthetic system consists of three distinct NRPSs, namely, DptA (684 kDa), DptBC (815 kDa), and DptD (265 $\mathrm{kDa}$ (87). In contrast, the closely related A54145 biosynthetic system comprises four NRPSs (LptA, LptB, LptC, and LptD) (86). It is assumed that DptBC arises from a fusion of two NRPSs similar to LptB and LptC. Finally, the nonribosomal

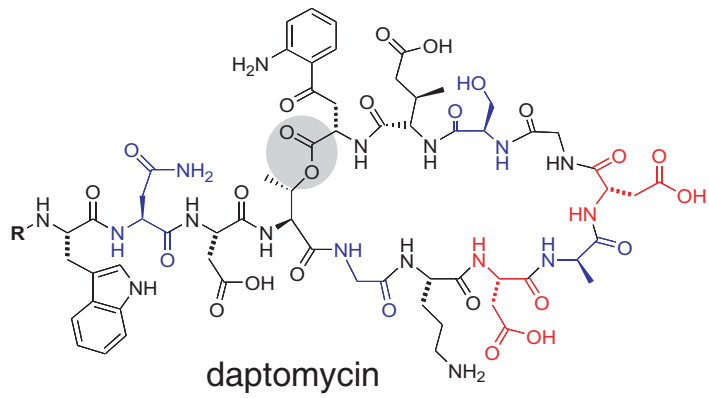

\begin{tabular}{|c|l|}
\hline factor & \multicolumn{1}{|c|}{$\mathbf{R}$} \\
\hline $\mathrm{A}_{21978 \mathrm{C}-\mathrm{C}_{1}}$ & 8-methyldecanoyl $\left(a \mathrm{C}_{11}\right)$ \\
\hline $\mathrm{A}_{21978 C} \mathrm{C}_{2}$ & 10-methylundecanoyl $\left(i \mathrm{C}_{12}\right)$ \\
\hline $\mathrm{A}_{12} 1978 \mathrm{C}-\mathrm{C}_{3}$ & 10-methyldodecanoyl $\left(a \mathrm{C}_{13}\right)$ \\
\hline daptomycin & n-decanoyl $\left(n \mathrm{C}_{10}\right)$ \\
\hline
\end{tabular}

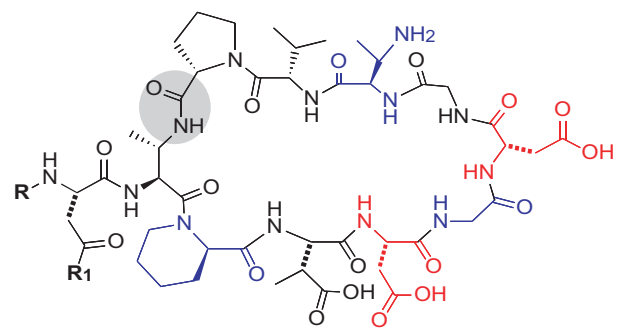

friulimicin / amphomycin

\begin{tabular}{|l|l|l|}
\hline \multicolumn{1}{|c|}{ factor } & $\mathbf{R}$ & $\mathbf{R} 1$ \\
\hline friulimicin $\mathrm{A}$ & $\triangle$ 3-isotridecenoyl $\left(i \mathrm{C}_{13}\right)$ & $\mathrm{NH}_{2}$ \\
\hline friulimicin $\mathrm{B}$ & $\Delta$ 3-isotetradecenoyl $\left(i \mathrm{C}_{14}\right)$ & $\mathrm{NH}_{2}$ \\
\hline friulimicin $\mathrm{C}$ & $\Delta$ 3-anteisotridecenoyl $\left(a \mathrm{C}_{13}\right)$ & $\mathrm{NH}_{2}$ \\
\hline friulimicin D & $\Delta$ 3-anteisopentadecenoyl $\left(a \mathrm{C}_{15}\right)$ & $\mathrm{NH}_{2}$ \\
\hline amphomycin A-1437 A & $\Delta$ 3-isotridecenoyl $\left(i \mathrm{C}_{13}\right)$ & $\mathrm{OH}$ \\
\hline amphomycin A-1437 B & $\triangle$ 3-isotetradecenoyl $\left(i \mathrm{C}_{14}\right)$ & $\mathrm{OH}$ \\
\hline amphomycin A-1437 E & $\triangle$ 3-anteisotridecenoyl $\left(a \mathrm{C}_{13}\right)$ & $\mathrm{OH}$ \\
\hline amphomycin A-1437 G & 3-anteisopentadecenoyl $\left(a \mathrm{C}_{15}\right)$ & $\mathrm{OH}$ \\
\hline
\end{tabular}

CDA biosynthetic system is a multienzyme complex consisting of three enzymatic subunits, CdaPS1 (799 kDa), CdaPS2 (395 $\mathrm{kDa})$, and CdaPS3 (259 kDa) (51).

The multifunctional NRPSs of daptomycin, A54145, and CDA are organized into sets of repetitive catalytic units called modules (Fig. 3). Each module is responsible for the specific incorporation of one residue into the peptide backbone (107). Therefore, the number of modules within the NRPSs exactly matches the number of residues of the corresponding peptides. Moreover, the order of modules corresponds directly to the primary sequence, because nonribosomal peptide synthesis proceeds colinearly in an $\mathrm{N}$-terminal-to-C-terminal direction 


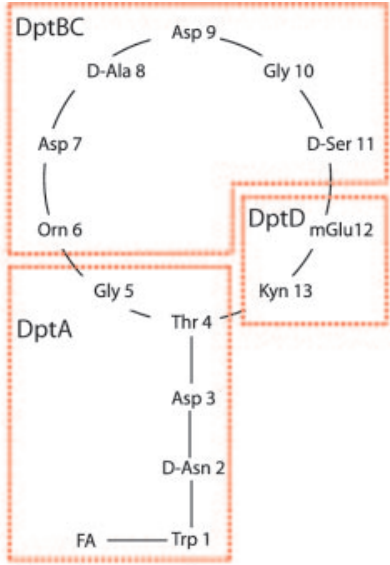

daptomycin

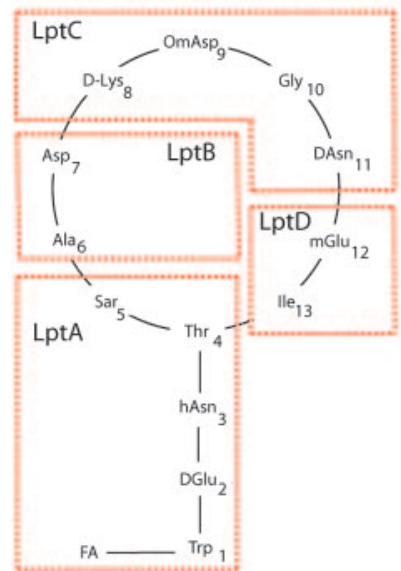

A 54145

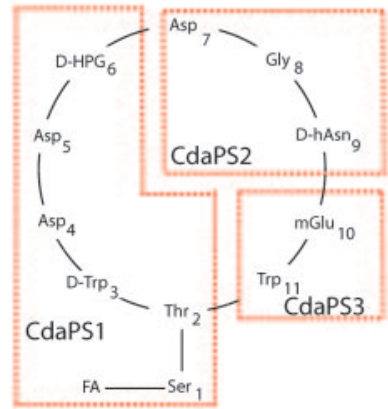

CDA
$\operatorname{DptA}(684 \mathrm{kDa})$

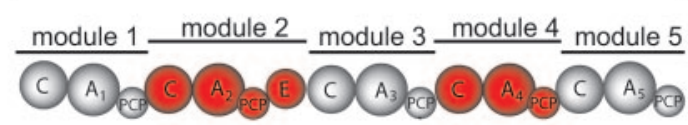

DptBC $(815 \mathrm{kDa})$

module 6 module 7 module 8 module 9 module 10 module 11

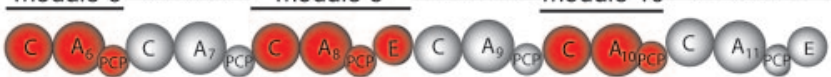

$\operatorname{DptD}(265 \mathrm{kDa})$

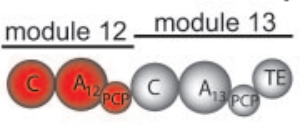

LptA

LptB

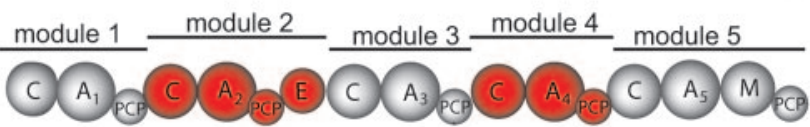

module 6 module 7

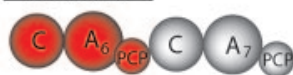

LptD

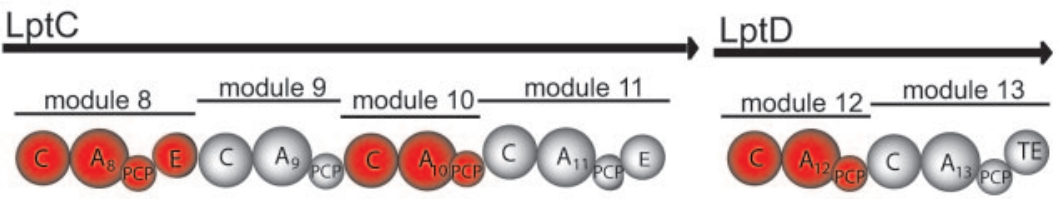

CdaPS1 $(799 \mathrm{kDa})$
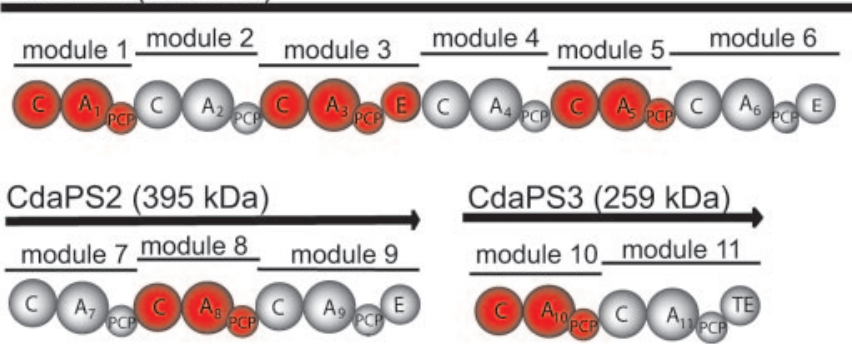

FIG. 3. Comparison of enzymatic subunits of the daptomycin (DptA, DptBC and DptD), A54145 (LptA, LptB, LptC, and LptD), and CDA (CDAI, CDAII and CDAIII) NRPSs that are responsible for the synthesis of the respective peptide cores. Parts of the peptide cores that are synthesized by their dedicated enzymatic subunits are surrounded by red dotted lines. The modules indicated in red and white are subdivided into catalytically independent domains responsible for substrate recognition/activation. FA, fatty acid; hAsn, 3-hydroxyasparagine; mGlu, 3-methylglutamate; Sar, sarcosine; omAsp, 3-methoxyaspartate; Orn, ornithine; Kyn, kynurenine.

(91). Such biosynthetic templates are also referred to as linear NRPSs (type A). In contrast to that, iterative NRPSs (type B) use their modules or domains more than once in the assembly of peptides that consist of repeated smaller sequences. Finally, nonlinear NRPSs (type C) constitute a considerable fraction of the NRPS repertoire where the sequence of the product does not directly correspond to the linear arrangement of modules and domains within the biosynthetic template. These various biosynthetic strategies of nonribosomal peptide synthesis were extensively reviewed by Mootz et al. (91).

The proper coordination of communication between partner NRPSs in trans (i.e., the last module of DptA and first module 


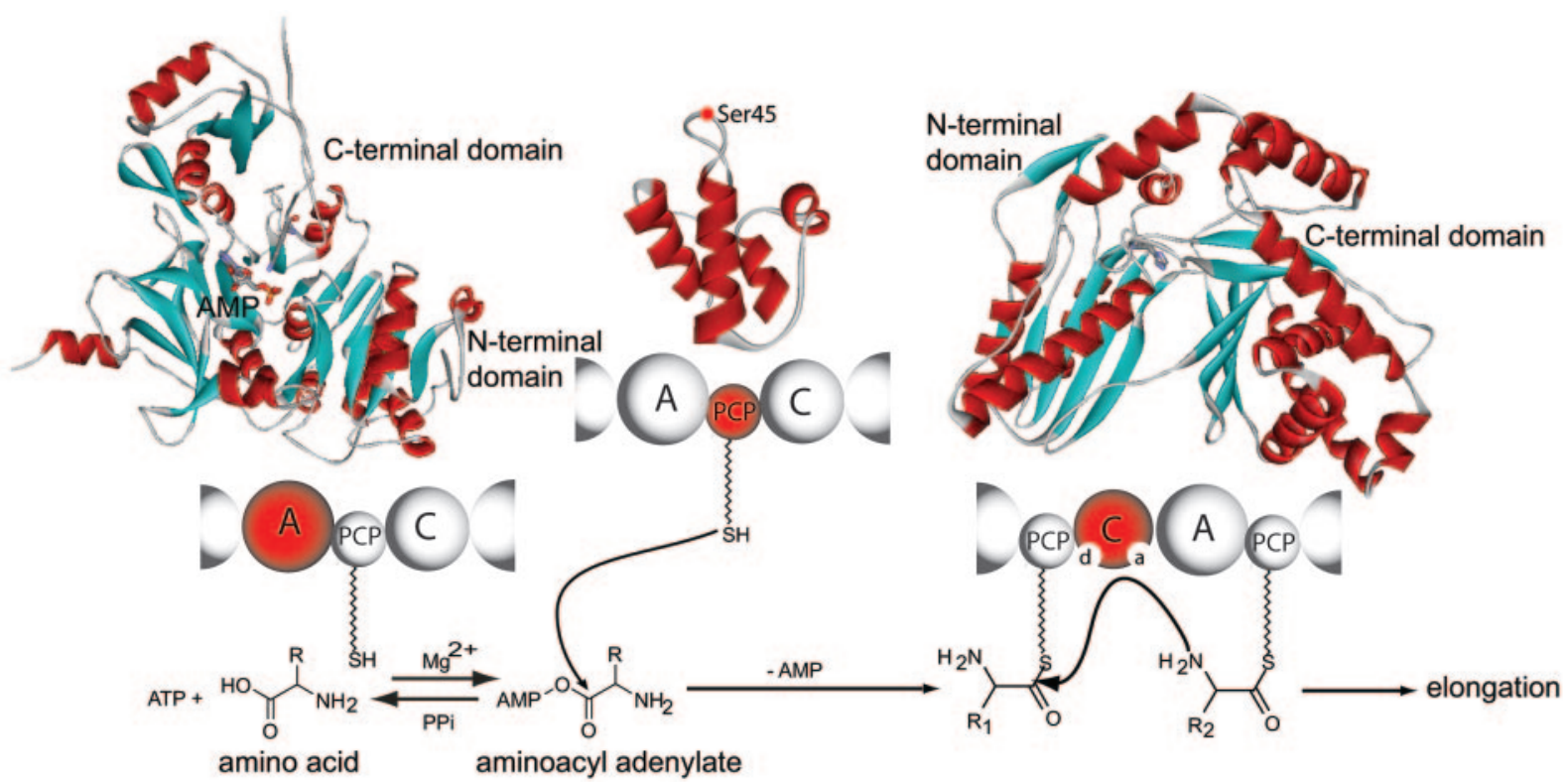

FIG. 4. Chemical principles of nonribosomal peptide synthesis. Domains in action are indicated in red and the respective crystal structures are shown above. First, the A-domain specifically recognizes a dedicated amino acid and catalyzes formation of the aminoacyl adenylate under consumption of ATP. Second, the activated aminoacyl adenylate is tethered to the free thiol group of the PCP-bound phosphopantetheine (ppan) cofactor. Third, the C-domain catalyzes peptide elongation. Here, the nucleophilic amine of the acceptor substrate nucleophilically attacks the electrophilic thioester of the donor substrate (a, acceptor site; d, donor site). The crystal structure of the A-domain is derived from the Phe-activating A-domain (PheA) of the first module of gramicidin S synthetase of B. brevis (22). The NMR-structure of the PCP is derived from the third module of the B. brevis tyrocidine synthetase (141), and the C-domain is derived from the crystal structure of VibH, a stand alone C-domain of the $V$. cholerea vibriobactin synthetase (60).

of DptBC) is facilitated by short regions at the $\mathrm{C}$ and $\mathrm{N}$ termini of the corresponding proteins (47). These communication-mediating (COM) domains, also referred to as docking domains, comprise 15 to 30 amino acid residues and prevent undesired interactions between mismatching NRPSs (i.e., the last module of DptA and first module of DptD), which would lead to the formation of truncated peptide products. Sequence alignments revealed that the overall identity among COM domains is low, reflecting the high degree of specialization for their dedicated partner COM domains. The first structural insights into the interaction between multimodular subunits were gained from NMR spectroscopy on related polyketide synthases (PKS) (14). Studies of fused docking domains of the 6-deoxyerythronolide B synthase (DEBS) multienzyme subunits DEBS2 and DEBS3 revealed that protein-protein recognition is primarily mediated by interhelical contacts. The most important determinant of docking is a set of conserved hydrophobic interactions between four $\alpha$-helices, which together form the core of a parallel four-helix bundle. In addition to the hydrophobic interface, two partially buried salt bridges between two of these $\alpha$-helices may play a role in stabilizing this docking interaction. Furthermore, such ionic contacts might contribute to the destabilization of misdocked partner PKS subunits. Knowledge of the structural aspects of intersubunit communication may contribute to engineering of optimized protein-protein interfaces between NRPS, PKS, and mixed NRPS/PKS systems.

NRPS modules are further subdivided into domains that catalyze the single reaction steps, such as amino acid activation, covalent binding of activated residues, amide bond formation, epimerization of covalently bound residues, and pep- tide release from the NRPS complex. These autonomous catalytic units will be discussed below.

\section{Dissecting the Modules into Domains}

At least three domains are necessary for the nonribosomal production of peptides (Fig. 4): the adenylation domain (Adomain), the peptidyl-carrier protein (PCP), and the condensation domain (C-domain). The A-domain ( $\sim 550$ aa) controls the first step of nonribosomal peptide synthesis, namely, the specific recognition and activation of the dedicated amino acid $(26,83)$. This domain catalyzes two reactions. First, the A-domain selects the cognate building block from the pool of available substrates, followed by activation as an aminoacyl adenylate intermediate (Fig. 4). The corresponding reaction in ribosomal synthesis is performed by aminoacyl-tRNA-synthetases, although these enzyme families share neither sequence nor structural relations (124). Two crystal structures of A-domains have been solved to date. These include the $\mathrm{Phe}_{1}$ activating A-domain (PheA) of the gramicidin S synthetase A of Bacillus brevis (22) (Fig. 4) and the 2,3-dihydroxybenzoate (DHB)-activating A-domain (DhbE) of Bacillus subtilis (83). They are composed of a large N-terminal subunit and a small C-terminal subunit. The active site is located at the junction between the two subunits. Comparison of the residues lining the active sites of PheA and DhbE and sequence alignments of other A-domains led to the identification of 10 residues that confer substrate specificity, also referred to as the codons of nonribosomal peptide synthesis (118). Using this nonribosomal 
code, it is possible to predict the substrate specificity of A-domains simply by sequence analysis.

Second, the activated aminoacyl adenylate is transferred onto the thiol group of the $4^{\prime}$-phosphopantetheine (ppan) cofactor of the PCP, which is the only NRPS domain without autonomous catalytic activity. The PCP ( $~ 80$ aa) facilitates the ordered transport of substrates and elongation intermediates to the catalytic centers with all intermediates covalently tethered to the 20 - $\AA$-long ppan cofactor (Fig. 4) $(34,116)$. This principle facilitates substrate channeling and overcomes diffusive barriers, therefore maximizing the catalytic efficiency of the NRPS-mediated biosynthesis (111). First insights into protein structure and function of PCPs were gained from an NMR study on PCP from Bacillus brevis tyrocidine synthetase (141) (Fig. 4). PCP exhibits a distorted four-helix bundle fold and an extended loop between the first two helices. An invariant Ser $_{45}$ residue, which serves as the site of ppan cofactor binding, is located at the interface between this loop and the second helix. The posttranslational apo-to-holo conversion of PCPs is catalyzed by NRPS associated 4 '-phosphopantetheinyl transferases, which use coenzyme A (CoA) as a substrate (72) (see "MANIPULATION OF CARRIER PROTEINS BY POSTTRANSLATIONAL MODIFICATION").

Formation of the peptide bond in nonribosomal peptide biosynthesis is mediated by the C-domain ( 450 aa) $(9,117)$. This domain catalyzes the nucleophilic attack of the downstream PCP-bound amino acid with its $\alpha$-amino group on the electrophilic thioester of the upstream PCP-bound amino acid or peptide (Fig. 4). The directionality of this process is realized by donor and acceptor sites on the C-domain for electrophiles and nucleophiles, respectively (91). According to the multiple carrier thio-template mechanism (121), the acceptor site binds the nucleophile with high affinity until the incoming electrophile completes the condensation process. First structural insights into this class of enzymes were gained from the crystal structure of the freestanding $\mathrm{C}$-domain $\mathrm{VibH}$ of the Vibrio cholerae vibriobactin synthetase (60) (Fig. 4). Pseudodimeric $\mathrm{VibH}$ consists of a C-terminal domain and an N-terminal domain, with each domain being an $\alpha \beta \alpha$ sandwich. The substrates DHB and norspermidine enter the active site, which is located at the interface of the two domains, from opposing sites of the C-domain, the so-called $\mathrm{N}$ - and C-faces. Therefore, these two faces would correspond to the assumed donor (Cface) and acceptor sites ( $\mathrm{N}$-face) of the $\mathrm{C}$-domain, respectively. Biochemical characterization of different $\mathrm{C}$-domains revealed that the acceptor site discriminates against amino acids of opposite stereochemistry and with noncognate side chains (7, 21). In contrast, the donor site is more tolerant of the respective electrophile. Nevertheless, further investigations with the $\mathrm{C}$-domain of tyrocidine elongation module 5 indicated that the donor position exhibits stereoselectivity toward the C-terminal residue for condensation reactions (21). This shows that, in addition to A-domains, C-domains serve as a selectivity filter in nonribosomal peptide synthesis.

\section{Proofreading of Nonribosomal Peptide Synthesis}

The low substrate specificity of ppan transferases causes undesired misacylation of PCPs. Since the bacterial cell produces a large fraction of CoA in the form of acyl-CoAs (53), it
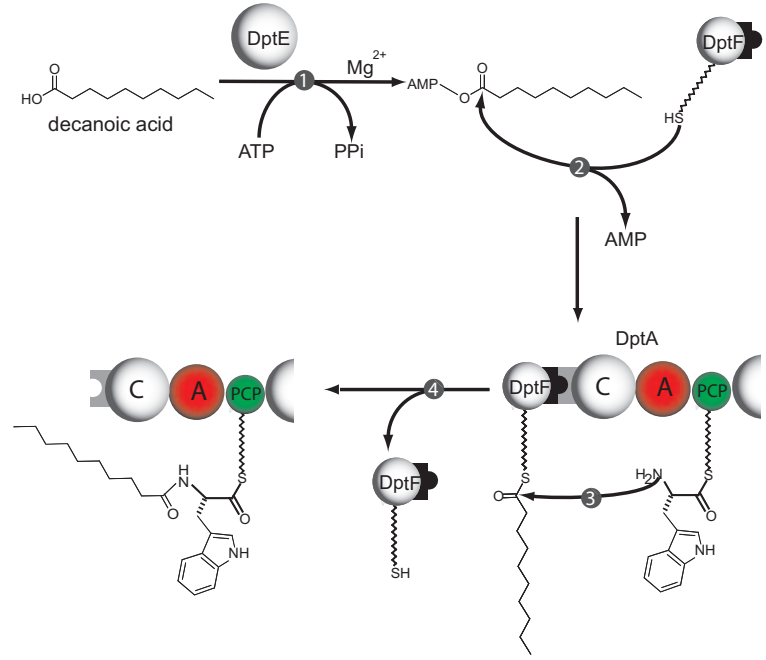

FIG. 5. Proposed mechanism of the lipidation of daptomycin. Decanoic acid is activated as decanoyl-adenylate under the consumption of ATP (1). This step is catalyzed by DptE. The fatty acid is transferred on to the ppan cofactor of the putative acyl-carrier protein $\mathrm{DptF}(2)$. DptF interacts with the starter C-domain of DptA, which catalyzes the subsequent acylation of $\operatorname{Trp}_{1}$ (3). Finally, DptF is released (4).

is therefore likely that these enzymes also modify the PCPs of NRPSs with acylated ppan cofactors. Such misprimed PCPs are not recognized by later-acting domains, thereby blocking nonribosomal peptide synthesis. In order to regenerate these misprimed NRPS templates, a type II thioesterase (TEII) is assumed to catalyze hydrolysis of the undesired acyl groups (108). Moreover, a recent study suggests that the TEII also hydrolyzes incorrectly loaded amino acids that are not processed by the nonribosomal machinery (148). According to this model, TEII discriminates "correct" from "incorrect" residues based on the increased half-life of unprocessed aminoacyl-Sppan intermediates. In contrast to this, TEII does not catalyze the hydrolysis of stalled peptide intermediates, which indicates that the release of these energy-consuming intermediates is prevented by rigorous editing of misloaded amino acids prior to incorporation into the product $(132,148)$.

\section{Lipidation of Non-Ribosomally Produced Peptides}

N-terminal lipidation is a key structural feature of many nonribosomal peptides, such as the acidic lipopeptide antibiotics, fengycin (Fig. 1), surfactin A (Fig. 1), syringomycin, and mycosubtilin, etc. As discussed above (see "DIVERSITY OF NONRIBOSOMAL PEPTIDES"), it is important for interaction with hydrophobic targets, e.g., cell membranes. However, in contrast to the well-studied peptide elongation, very little is known about the mechanism of this chemical transformation. In the case of daptomycin, the deduced translation products of the $d p t E$ and $d p t F$ genes are likely to have a role in $\mathrm{N}$-terminal lipidation (87). DptE exhibits conserved motifs typical of adenylate-forming enzymes and may therefore activate the longchain fatty acid as acyl-adenylate (Fig. 5). A similar mode of activation was previously described for the long-chain fatty acyl-AMP ligases of Mycobacterium tuberculosis (129). Accord- 
A)

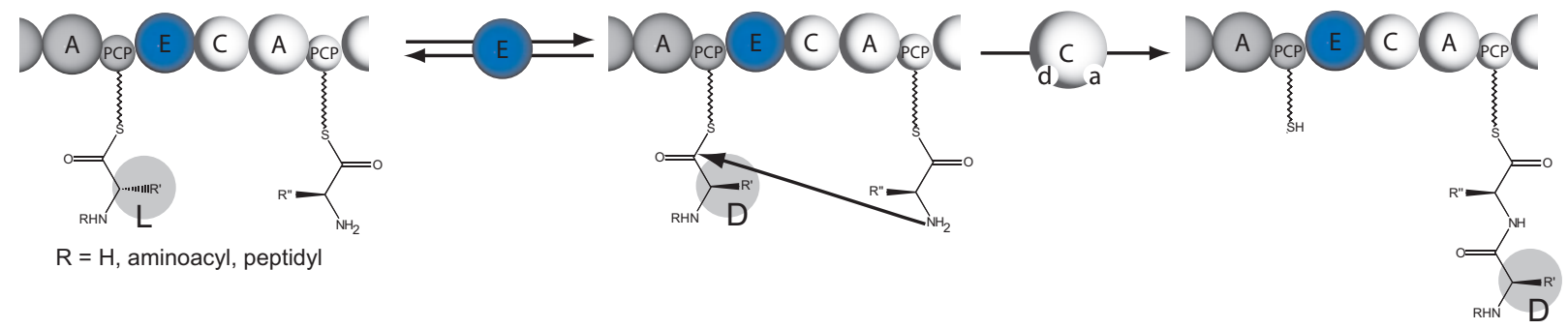

B)

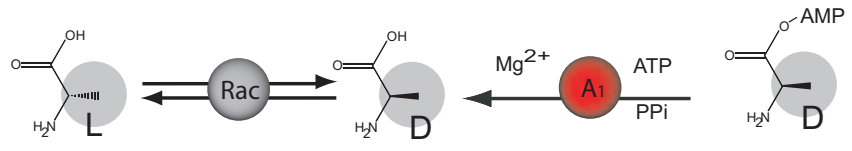

C)
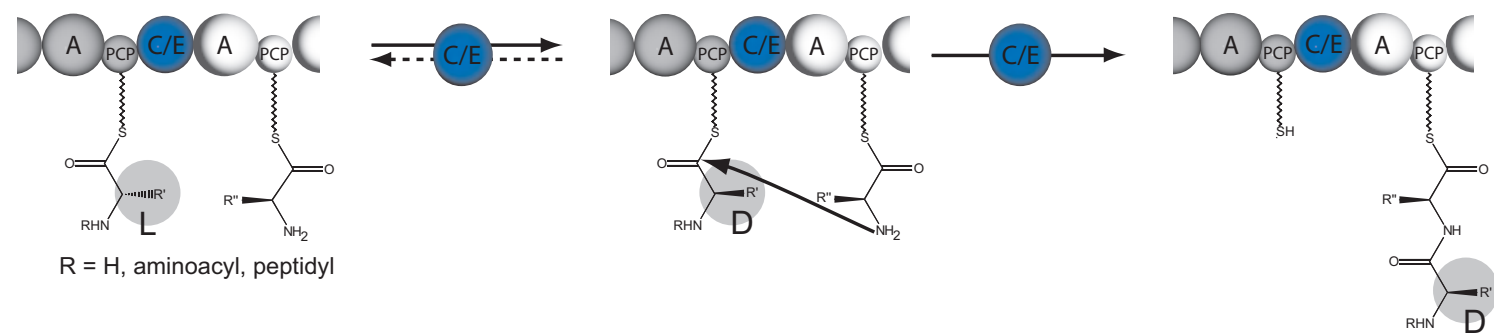

FIG. 6. Proposed mechanisms underlying amino acid epimerization. (A) The E-domain converts the PCP-tethered aminoacyl substrate into a $\mathrm{D} / \mathrm{L}$ equilibrium. The stereoselective donor site (d) of the C-domain of the downstream module uses only the D-configured amino acid for subsequent peptide elongation. (B) In some cases, an external racemase (Rac) catalyzes the racemization of a freely diffusible amino acid. Here, a stereoselective A-domain is the determinant that activates solely the corresponding D-enantiomer. (C) D-Amino acid incorporation into arthrofactin, syringomycin, and syringopeptin is catalyzed by a new type of condensation domain (C/E-domain). Epimerization does not take place unless the PCP downstream of this C/E-domain is loaded with the dedicated amino acid. It is not yet known whether the epimerization reaction is reversible or not. After epimerization of the upstream aminoacyl/peptidyl thioester, the C/E-domain mediates the elongation of the peptidyl chain with ${ }^{\mathrm{D}} \mathrm{C}_{\mathrm{L}}$ chirality.

ing to this work, long-chain fatty acids are activated as acyladenylates, which are then transferred onto the ppan cofactor of the N-terminal PCP of the corresponding PKS. However, the daptomycin biosynthetic system lacks such an N-terminal PCP. Instead, DptF may serve this function due to its significant alignment to ppan-binding acyl carrier proteins (ACPs). This domain could then transfer the ppan-bound fatty acid to $\operatorname{Trp}_{1}$ tethered to the N-terminal module of DptA. Acylation of $\operatorname{Trp}_{1}$ is presumably catalyzed by the most upstream $\mathrm{C}$-domain, the socalled starter C-domain. Specific starter C-domain-ACP docking may facilitate this acyl transfer reaction (Fig. 5). However, further studies are needed to clarify the specificity and biochemistry of the interaction between the ACP and the starter C-domain of the daptomycin as well as other lipopeptide-encoding biosynthetic systems.

\section{Generation of D-Amino Acid Residues in NRPSs}

One striking feature of many NRPSs is that they incorporate D-amino acids into their peptide products. The D-con- figured residues may inhibit the degradation of nonribosomal peptides by naturally L-specific proteases or may serve structural functions by determining the bioactive conformation $(70,79,120)$. In most cases, incorporation of $\mathrm{D}$-amino acids into the peptide sequence is mediated by an interplay between the epimerization domain (E-domain; 450 aa) $(95,120)$ and the downstream C-domain (Fig. 6A). The E-domain catalyzes racemization (equilibration between Land D-enantiomers) of the PCP-bound L-amino acid or epimerization of the C-terminal amino acid (equilibration between L- and D-epimers) of the growing peptide chain. In order to ensure selective incorporation of the D-amino acid into the peptide backbone, the donor site of the downstream C-domain is D-specific for the incoming cofactor-bound electrophile (7). Hence, the C-domain functions as a catalyst directing the condensation of an upstream D-amino acid with a downstream L-amino acid ( ${ }^{\mathrm{D}} \mathrm{C}_{\mathrm{L}}$ catalyst).

A different mechanism for the incorporation of $\mathrm{D}$-amino acids is utilized by the cyclosporine synthetase (Fig. 6B) (50). The corresponding biosynthetic gene cluster encodes an ala- 
nine racemase to provide substrate for the D-Ala-selective Adomain in the first module. This shows that besides C-domains, A-domains may also represent a stereoselective filter in nonribosomal peptide synthesis.

Recently, a third strategy of D-amino acid incorporation was observed in multiple gram-negative Pseudomonas strains producing arthrofactin, syringomycin, and syringopeptin (1). The lipopeptidolactone arthrofactin, for instance, contains seven D-amino acids, yet there are no E-domains in any of the three NRPSs, ArfA, ArfB, and ArfC. Moreover, kinetic measurements revealed that at least the three most upstream A-domains activate $\mathrm{L}$-amino acids rather than $\mathrm{D}$-amino acids. Interestingly, epimerization of amino acids is catalyzed by a new type of domain, a C/E-domain, which is proposed to have dual catalytic roles for epimerization and condensation (Fig. 6C). Remarkably, the epimerization reaction does not take place unless the PCP downstream of this C/E-domain is loaded with the dedicated amino acid. Therefore, the epimerization activity may be triggered by a conformational change of the $\mathrm{C} / \mathrm{E}$-domain which is induced by the aminoacylated downstream PCP that is primed for peptide bond formation. After epimerization of the upstream aminoacyl/peptidyl thioester, the $\mathrm{C} / \mathrm{E}$-domain finally catalyzes the elongation of the peptidyl chain with ${ }^{\mathrm{D}} \mathrm{C}_{\mathrm{L}}$ chirality.

\section{MACROCYCLIZATION CATALYZED BY NONRIBOSOMAL THIOESTERASE DOMAINS}

Nonribosomal peptides grow by consecutive addition of activated aminoacyl monomer units. The elongated chain is translocated each time from upstream to downstream PCPs during chain elongation. Once the peptide chain reaches its full length at the most downstream PCP, it has to be released in order to reactivate the NRPS machinery for the next synthesis cycle. Typically, termination of peptide synthesis is accomplished by a thioesterase domain (TE-domain; 280 aa) fused to the C-terminal module (68). This enzyme uses an active site serine as a nucleophilic catalyst. Peptide release is initiated by transfer of the ppan-bound peptide chain to the active site serine of the downstream TE-domain to generate an acyl$O$-TE intermediate (68). This covalent enzyme intermediate may break down either by the attack of a water molecule to yield a linear peptide (e.g., vancomycin) or by attack of an internal nucleophile, producing a cyclopeptide (e.g., daptomycin) (Fig. 7A).

While TE-domains represent the most common solution to peptide release in nonribosomal biosynthesis, alternative strategies are known. For instance, the most downstream C-domain of cyclosporine synthetase is proposed, in the synthesis of cyclosporine 7 (Fig. 1), to catalyze peptide release by head-to-tail condensation (Fig. 7B) (140). Moreover, peptide release can occur under reduction of the carboxy group mediated by the $\mathrm{NAD}(\mathrm{P}) \mathrm{H}$-dependent reduction domain (R-domain) such as in the biosynthesis of the linear peptide alcohol gramicidin $\mathrm{A}$ in B. brevis (62) and in the formation of the macrocyclic imine nostocyclopeptide 8 (Fig. 1) from Nostoc sp. (Fig. 7C) (6).

However, macrocyclization catalyzed by nonribosomal TEdomains seems to be the favored mechanism for peptide release, not least because of the role this structural constraint plays in resistance to proteolytic degradation and enhanced bioactivity. For example, the conformation of daptomycin is constrained by a branched cyclic decapeptide lactone derived from TE-mediated cyclization of an L-threonine side chain onto the $\mathrm{C}$ terminus (51). Considering the diversity in cyclization strategies of nonribosomal peptides, it is not surprising that the overall identity among TE-domains is only 10 to $15 \%$, therefore reflecting the high degree of specialization for their catalyzed cyclization reactions (106). Structural and mechanistic aspects of these versatile macrocyclization catalysts (also referred to as peptide cyclases) are discussed in the following section.

\section{Structural and Mechanistic Aspects of Peptide Cyclases}

First structural and mechanistic insights into the mode of TE-mediated peptide cyclization were gained from the crystal structure of the surfactin cyclase (Srf TE) (15). The crystallographic studies revealed similarities to structures previously solved for $\alpha / \beta$ hydrolase family members. However, the Srf TE most significantly differed from the canonical fold of this superfamily by an extended insertion composed of three $\alpha$-helices that reach over the active site. Based on alignment, this "lid" differs significantly from the corresponding regions of other TE domains, suggesting that the substrate specificity is encoded in this predominantly nonconserved region of the cyclase (68). The nonconserved residues in the lid may direct cyclization through specific interactions with the Srf TE-bound peptide chain. Based on further studies, the two positively charged residues $\mathrm{Lys}_{111}$ and $\mathrm{Arg}_{120}$ in the active site may also contribute to the proper folding of the substrate by coordination of the negatively charged residues $\mathrm{Glu}_{1}$ and $\mathrm{Asp}_{5}$ in the surfactin sequence (130).

In NRPS assembly lines, the TE-domain acts in concert with the upstream PCP that donates the ppan-bound peptide chain. In the case of Srf TE, a putative interaction site allows docking of the $\mathrm{C}_{\alpha}$ chain of PCP to the cyclase (15). The peptide chain tethered to the 20 - $\AA$-long ppan cofactor is presumably directed via a cleft into the active site of the globular cyclase and transferred onto a conserved serine residue. This residue belongs to a catalytic triad composed of $\mathrm{Ser}_{80}, \mathrm{His}_{207}$, and $\mathrm{Asp}_{107}$. Cocrystallization studies with a boronic acid inhibitor revealed distinct recognition and binding of the $\mathrm{C}$-terminal residues $\mathrm{Leu}_{7}$ and $\mathrm{D}-\mathrm{Leu}_{6}$ of the surfactin peptide in the active site (130). Finally, breakdown of the generated acyl-O-TE intermediate occurs by regioselective intramolecular attack of the fatty acid $\beta$-hydroxyl group on the oxoester bond to exclusively release the macrolactone.

\section{Autonomous Cyclization Activity of Excised TE-Domains}

The great pharmacological potential of many cyclic peptides emphasizes their role in drug discovery, as they show specific interactions with defined cellular targets and high stability against proteolytic digestion (111). They are therefore very promising scaffolds for drugs. So far, modern organic chemistry faces many difficulties in the reliable production of cyclopeptides. In many cases, the yield is poor or the reaction lacks sufficient regio- and stereoselectivity $(23,109)$. These problems could be solved by using nonribosomal cyclases, which catalyze the regio- and stereoselective cyclization of linear precursor peptides without the use of protecting groups. However, the 

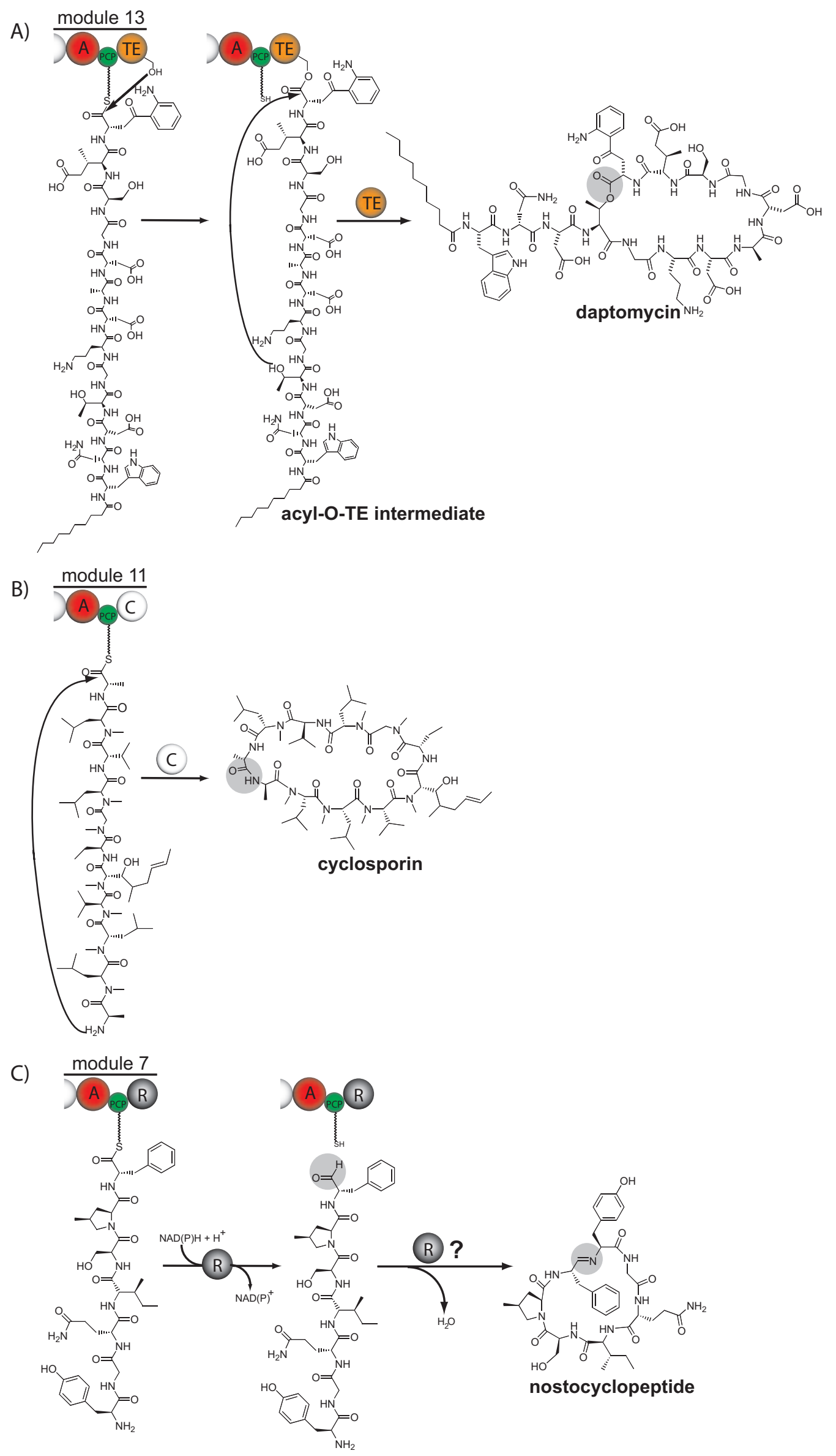


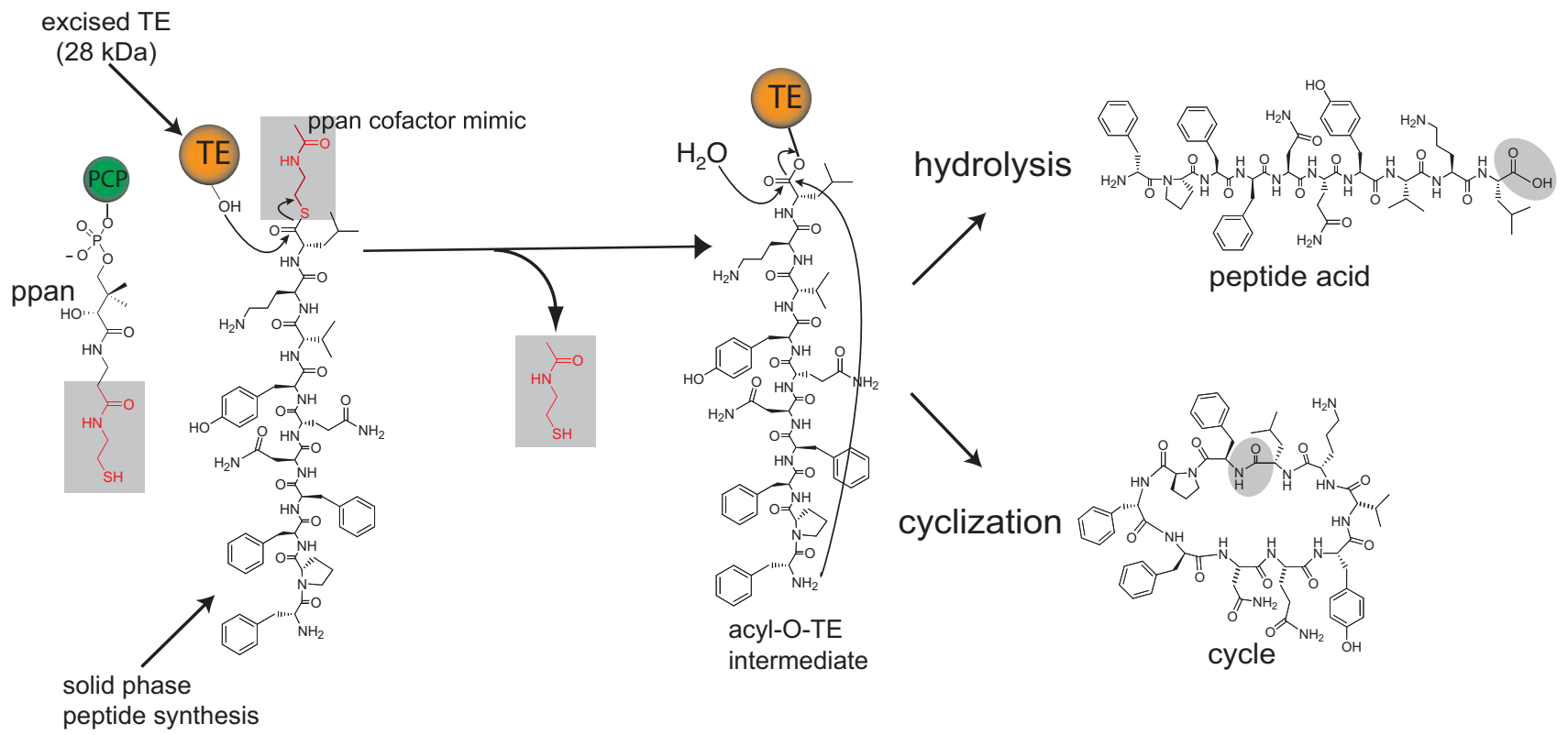

FIG. 8. The experimental design for the study of excised cyclases exemplified for Tyc-TE. First, the NRPS multienzyme machinery for tyrocidine synthesis is replaced by solid-phase peptide synthesis. Second, the TE-domain is used as an excised enzyme for in vitro peptide cyclization. Third, recognition of the artificial substrate by the excised cyclase is ensured by the phosphopantetheine cofactor mimic SNAC (highlighted by shading).

application of nonribosomal TE-domains for cell-free synthesis of cyclic peptides requires translation between the biological and chemical languages. First, the complex NRPS multienzyme machinery required for peptide elongation is replaced by well-established solid-phase peptide synthesis (SPPS), which greatly facilitates the rapid synthesis of peptides containing unnatural amino acids (109). Second, the TE-domain is used as an isolated enzyme for in vitro peptide cyclization, because the large size of the whole multienzyme complex causes severe preparative problems. Third, to ensure acylation of the excised TE-domain, the natural PCP-bound phosphopantetheine prosthetic group is replaced by a cofactor mimic, which is attached to the C-terminal end of the chemically synthesized peptide.

This chemoenzymatic approach was first achieved by a cooperation between the Walsh and Marahiel laboratories, which reported on the isolation and characterization of the TE-domain of tyrocidine synthetase from Bacillus brevis (Fig. 8) (127). Incubation of a chemically synthesized tyrocidine decapeptidyl- $N$-acetylcysteamine (SNAC) thioester and excised tyrocidine cyclase (Tyc TE) resulted in the formation of the cyclic decapeptide antibiotic tyrocidine A (Fig. 1). Hydrolysis of the substrate mimic could be detected to a lesser extent and might be due to the fact that the excised cyclase lacks the hydrophobic environment of the multienzyme complex. Recent results indicate that the interaction of the isolated Tyc TE with detergent micelles may serve to mimic the natural contacts of this domain with the larger synthetase (149). In fact, the addition of nonionic detergent induced a significant shift in the product ratio of Tyc TE in favor of macrocyclization.

To explore the substrate specificity of Tyc TE, a scan through all 10 positions of the peptidyl-SNAC thioester was performed (127). Notably, it was found that only the substitution of amino acids near the end of the decapeptide, namely, D-Phe ${ }_{1}$ and $\mathrm{L}_{-} \mathrm{Orn}_{9}$, significantly decreased the rate of TEcatalyzed cyclization. It was also observed that thioester substrates 6 to 14 residues in length could be efficiently cyclized by Tyc TE, resulting in the formation of different-size macrolactams (67). Alterations of the peptide backbone either by the replacement of three amino acid blocks with flexible spacers or by the replacement of individual amide bonds with ester bonds provided evidence that product-like intramolecular hydrogen bonds facilitate peptide preorganization (128). This preorganization was efficient enough to allow macrolactone formation by using a hydroxyl group as intramolecular nucleophile despite the lower nucleophilicity of hydroxyl compared to amine. Based on these findings, a model of a minimal cyclization substrate for the Tyc TE was postulated (128).

FIG. 7. Macrocyclization strategies. (A) The tridecapeptidyl chain of daptomycin tethered to the ppan cofactor of the most downstream PCP is transferred to an active site serine of the TE-domain forming the acyl-O-TE intermediate. Subsequent product release is carried out by the attack of an internal nucleophile $\left(\mathrm{L}-\mathrm{Thr}_{4}\right)$ on the oxoester bond to give the cyclic branched macrolactone. (B) Head-to-tail macrolactamization of the undecapeptide cyclosporine is catalyzed by the most downstream C-domain. Mechanistic details are still unknown. (C) Macrocyclic imine formation of nostocyclopeptide. First, the C-terminal residue of the ppan-tethered peptide is reduced by the action of an NAD(P)H-dependent $\mathrm{R}$-domain to give an aldehyde, which is intramolecularly captured by the $\mathrm{N}$ terminus to give a macrocyclic imine. Future research will show if the R-domain also mediates this final macrocyclization step. 


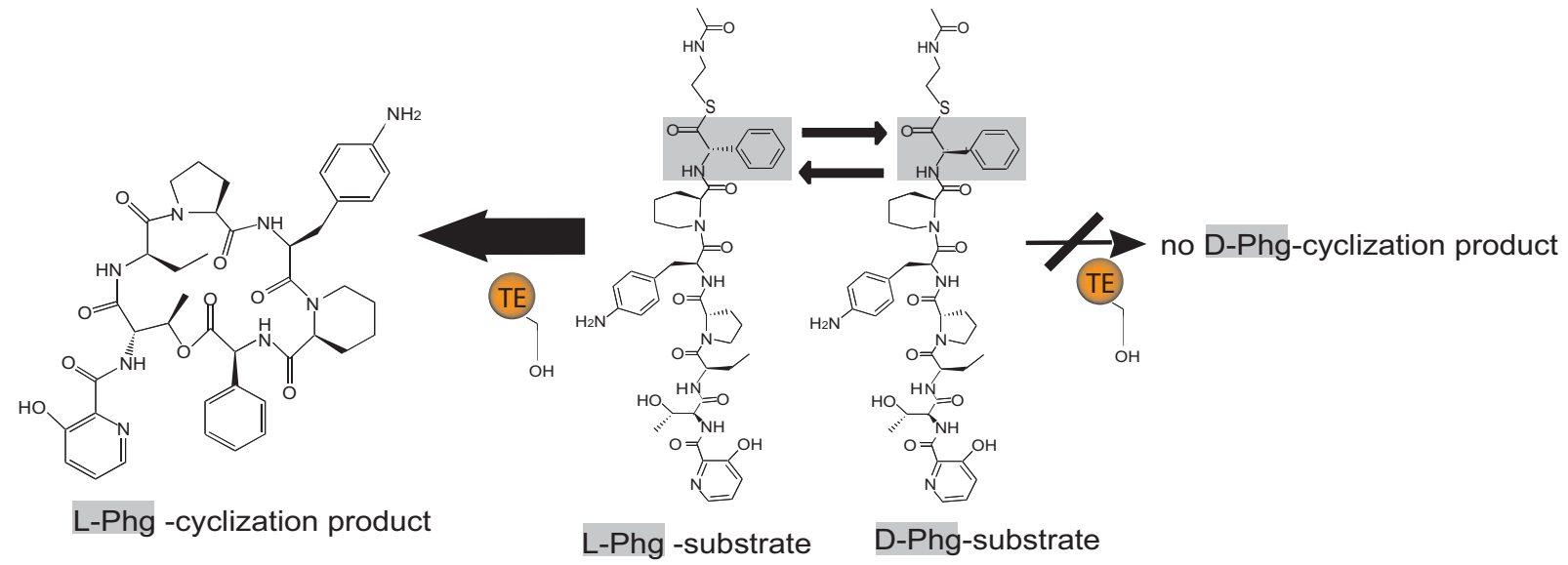

\section{in situ substrate racemization}

FIG. 9. Dynamic kinetic resolution of a streptogramin B SNAC substrate with Phg at the C terminus, which is prone to in situ substrate racemization. The resulting two diastereomers are able to acylate the active Ser residue of SnbDE TE resulting in two different peptidyl- $O$-TE intermediates. However, only the peptidyl- $O$-TE intermediate with the L-Phg-configuration is able to undergo cyclization to the natural product with L-Phg configuration.

\section{Generality of TE-Catalyzed Peptide Cyclization}

To provide evidence for the general utility of TE catalysis as a means to synthesize a wide range of macrocyclic compounds, peptide cyclases from other NRPS systems were cloned and overexpressed. The recombinant thioesterase domain SnbDE TE from Streptomyces pristinaespiralis is a versatile cyclase for the production of streptogramin $\mathrm{B}$ antibiotics such as pristinamycin (Fig. 1) (80). Although the streptogramin $B\left(S_{B}\right)$ SNAC substrates with the natural phenylglycine $(\mathrm{Phg})$ at the $\mathrm{C}$ terminus undergo rapid C-terminal racemization under assay conditions, stereoselective SnbDE TE only incorporates L-Phg into the cyclic product (Fig. 9). This dynamic kinetic resolution (131) simplifies challenging $S_{B}$ synthesis to standard peptide chemistry and subsequent enzymatic reaction. Besides having high stereoselectivity, SnbDE TE was able to mediate both macrolactonization and macrolactamization of peptide thioester substrates. Interestingly, macrolactamic $S_{B}$ derivatives are promising pharmacophores because in some cases, $\mathrm{S}_{\mathrm{B}}$ resistance arises from lyase-catalyzed cleavage of the natural lactone bond (92).

In addition to providing insights into stereoselectivity, biochemical studies of the recombinant $S$. coelicolor CDA TE have provided important insights into the regioselectivity of peptide cyclases. Incubation of N-terminally acetylated CDA thioester analogs with CDA TE resulted in two regioisomeric macrolactones which arise from simultaneous nucleophilic attack of the two adjacent $\mathrm{Thr}_{2}$ and $\mathrm{Ser}_{1}$ residues onto the C-terminal $\operatorname{Trp}_{11}$ of the acyl-enzyme intermediate (45). This relaxed regioselectivity was used to rationally manipulate the ring size of the macrocyclic product. For instance, substitution of either $\mathrm{Thr}_{2}$ or Ser $\mathrm{S}_{1}$ by Ala led to selective formation of a decapeptide or undecapeptide lactone ring. Interestingly, elongation of the $\mathrm{N}$-terminal acyl group by four methylene groups to the natural length $\left(\mathrm{C}_{6}\right)$ of $\mathrm{CDA}$ turned the relaxed regioselectivity into a strict regioselectivity, yielding solely the decapeptide lactone ring derived from cyclization via $\mathrm{Thr}_{2}$. This result suggests the crucial role of the lipid chain in controlling the regioselectivity of TE-mediated macrocyclization. Binding of the N-terminal fatty acid in the active site of CDA TE might ensure a precise positioning of the $\mathrm{Thr}_{2}$ residue required for a regioselective attack onto the acyl- $O$-TE oxoester.

To further expand the set of cyclization catalysts, the peptide cyclases Syr TE from syringomycin synthetase, Fen TE from fengycin synthetase, and Myc TE from mycosubtilin synthetase were cloned and overexpressed $(112,113)$. However, the in ability to recognize and bind conventional peptidyl-SNAC substrates precluded examination of these cyclases. To mimic the natural substrate presentation as close as possible, a strategy which allowed Sfp-catalyzed loading of peptidyl-CoA substrates onto apo-PCP-TE didomains was employed (113). This strategy takes advantage of the direct interaction between the ppan-bound substrate of the PCP and the C-terminally adjacent TE-domain. Using this approach, it was possible to detect cyclization of a linear fengycin analog. However, one major drawback of this method is that the ppan cofactor remains attached to the PCP-TE didomain, thereby blocking Sfp-catalyzed transfer of additional peptidyl-CoA substrates onto PCP. To force multiple turnover catalysis, reloading of the ppanPCP-TE didomain was attempted by chemical transthioesterification using peptidyl-thiophenol substrates (112). Surprisingly, instead of ppan reloading, the highly electrophilic peptidyl-thiophenol substrates directly acylated the TE active site serine. Furthermore, it was possible to biochemically characterize Syr TE, Fen TE, and Myc TE, which displayed no activity with less electrophilic peptidyl-SNAC substrates.

Solid-phase peptide synthesis enables the detailed analysis of NRPS-derived peptide cyclases. However, relatively little is known about the substrate specificity of macrocyclization catalysts of mixed NRPS/PKS biosynthetic systems. Biochemical studies of such systems are still hampered due to the challenges involved in synthesizing suitable linear precursor compounds. Nevertheless, researchers have begun to explore the substrate 
tolerances of the epothilone $\mathrm{C}$ and cryptophycin terminal TEdomains that mediate macrolactonization of mixed NRPS/ PKS-derived chain elongation intermediates $(5,12)$. In the former case, the artificial linear substrate was generated from the parent compound epothilone $\mathrm{C}$ via hydrolytic ring opening and subsequent conversion of the free acid into the SNAC thioester. Treatment of this thioester with epothilone TE generated a mixture of the 16-membered macrolactone, epothilone $\mathrm{C}$, and the hydrolysis product, seco-epothilone $\mathrm{C}$ (12). In contrast, no conversion of the SNAC thioester to epothilone C was detected in high-performance liquid chromatography assays in the absence of the recombinant TE-domain. Similarly, the isolated TE-domain from the cryptophycin biosynthetic pathway was capable of generating 16-membered depsipeptide rings with high efficiency (5). While epothilone TE was probed with only one substrate, a monomer-based chemical synthesis approach allowed for the characterization of cryptophycin TE with various SNAC substrates. These studies revealed considerable tolerance for structural variation within the seco-cryptophycin unit $\mathrm{C} \beta$-alanine residue, whereas a terminal phenyl ring in unit $\mathrm{A}$ is essential for efficient cyclization. These investigations are likely to provide access to novel compounds by combining synthetic chemistry and mixed NRPS/PKS metabolic enzymes.

\section{Chemoenzymatic Approaches toward Novel Cyclopeptides}

In order to investigate the general utility of NRPS cyclases for generating small molecules with different therapeutic potential, broad substrate tolerance is highly desirable. Kohli and coworkers showed that Tyc TE was capable to cyclize peptide substrates, in which up to 7 of 10 cognate residues were simultaneously replaced (66). Macrolactamization of these linear peptide precursors containing an integrated RGD sequence yielded potent inhibitors of ligand binding by integrin receptors, with cyclization and $\mathrm{N}$-methylation being important contributors to nanomolar potency (Fig. 10). Therefore, the therapeutic activity of the cyclization product was successfully moved from infectious disease (tyrocidine A) to cardiovascular pharmacology. The ability of Tyc TE to tolerate simultaneous side chain alterations was further utilized to mediate cyclization of substrates containing nonpeptidic elements. Incorporation of $\varepsilon$-amino acid building blocks into the peptide backbone led to the formation of cyclic polyketide/tyrocidine hybrids (Fig. 10) (65), which could be used to further optimize macrocyclic peptide/polyketide natural products, such as the immunosuppressant rapamycin and the anticancer agent epothilone (31). Furthermore, the insertion of $(E)$-alkene-dipeptide isosters allows the peptide backbone to be modified postsynthetically by chemical metathesis (40).

To evaluate the potential utility of excised TE domains for generating cyclic peptide libraries, a combinatorial approach was developed by Walsh and coworkers (69). In a biomimetic synthetic strategy, a solid-phase PEGA [poly(ethylene glycol)acrylamide copolymer] resin functionalized with a synthetic tether substitutes for the ppan cofactor of the PCP (Fig. 10). Subsequent SPPS was used for the preparation of more than 300 linear tyrocidine derivatives. When these solid-supportbound peptides were incubated with the recombinant Tyc TE, the cyclase could productively catalyze peptide release by enzymatic on-resin cyclization. The resulting library of cyclopep- tides revealed that replacement of D-Phe ${ }_{4}$ in tyrocidine A (Fig. 1) by a positively charged D-amino acid led to 30 -fold selectivity for bacterial membranes, thereby minimizing the hemolysis of red blood cells. These improved tyrocidine derivatives can now be translated back into an engineered NRPS template for large scale production via fermentation.

The chemoenzymatic potential of Tyc TE was also used to generate glycosylated cyclopeptides. Using this cyclase, macrocyclized tyrocidine decapeptide analogs with unnatural propargylglycine residues incorporated at positions 3 to 8 were prepared (74). The peptide backbones containing these alkyne residues allowed subsequent postsynthetic modification to selectively introduce azido-functionalized sugar residues by copper(I)-mediated $[3+2]$ cycloaddition reactions, also referred to as "click chemistry" (Fig. 11A). Later, Lin and coworkers developed an alternative method to prepare glycosylated cyclopeptides by incorporating glycosylated amino acids into linear peptides via SPPS followed by enzyme-catalyzed macrolactamization (Fig. 11B) (73). Numerous O-linked glycosylated peptidolactams were prepared using glycosylated serine or tyrosine residues at positions 5 to 8 .

While conventional chemical glycosylation of cyclic peptides suffers from little regiochemical control and enzymatic glycosylation is limited by the high substrate specificity of glycosyltransferases, these chemoenzymatic strategies combine regioselective incorporation of sugar moieties with the broad tolerance of Tyc TE for side chain replacements. Hence, these approaches allow carbohydrate complexity to be generated into macrocyclic peptides and should be generalizable to other NRPS cyclases, thereby providing a powerful tool for the production of novel drug leads by large cyclic library screens.

Using chemoenzymatic peptide cyclization, it should also be feasible to make libraries of lipopeptides. For instance, the approved antibiotic daptomycin (see "DIVERSITY OF NONRIBOSOMAL PEPTIDES") is a complex lipopeptide to approach synthetically. Moreover, chemical modifications of this nonribosomal cyclopeptide have been restricted to the $\alpha$-amino group of L-Trp ${ }_{1}$ and the $\delta$-amino group of L-Orn 6 (25, $49,114)$. Interestingly, five of the amino acids in daptomycin's lactone ring are found at the same positions in CDA. In addition, both lipopeptides comprise decapeptide lactone rings. Therefore, the capability of CDA cyclase for the chemoenzymatic generation of daptomycin was investigated. Simultaneous incorporation of six daptomycin-specific residues into the CDA backbone and elongation of the $\mathrm{N}$ terminus by two residues yielded a daptomycin derivative which contained LAsn at position 2 and L-Glu at position 12 (44). In accordance with acidic lipopeptide antibiotics, the bioactivity of this chemoenzymatic assembled daptomycin analog is dependent on the presence of calcium ions (see "DIVERSITY OF NONRIBOSOMAL PEPTIDES”). To identify calcium-binding sites in the lipotridecapeptide chain, all four acidic residues were successively substituted by either Asn or Gln. Bioactivity studies revealed that only $\mathrm{Asp}_{7}$ and $\mathrm{Asp}_{9}$ are essential for antimicrobial potency (44). According to a recent NMR structure of daptomycin (101), both residues are part of a type II' $\beta$-turn at the anionic/polar end of the amphiphatic molecule. This structural feature is likely to be important for calcium binding and therefore biological activity. 


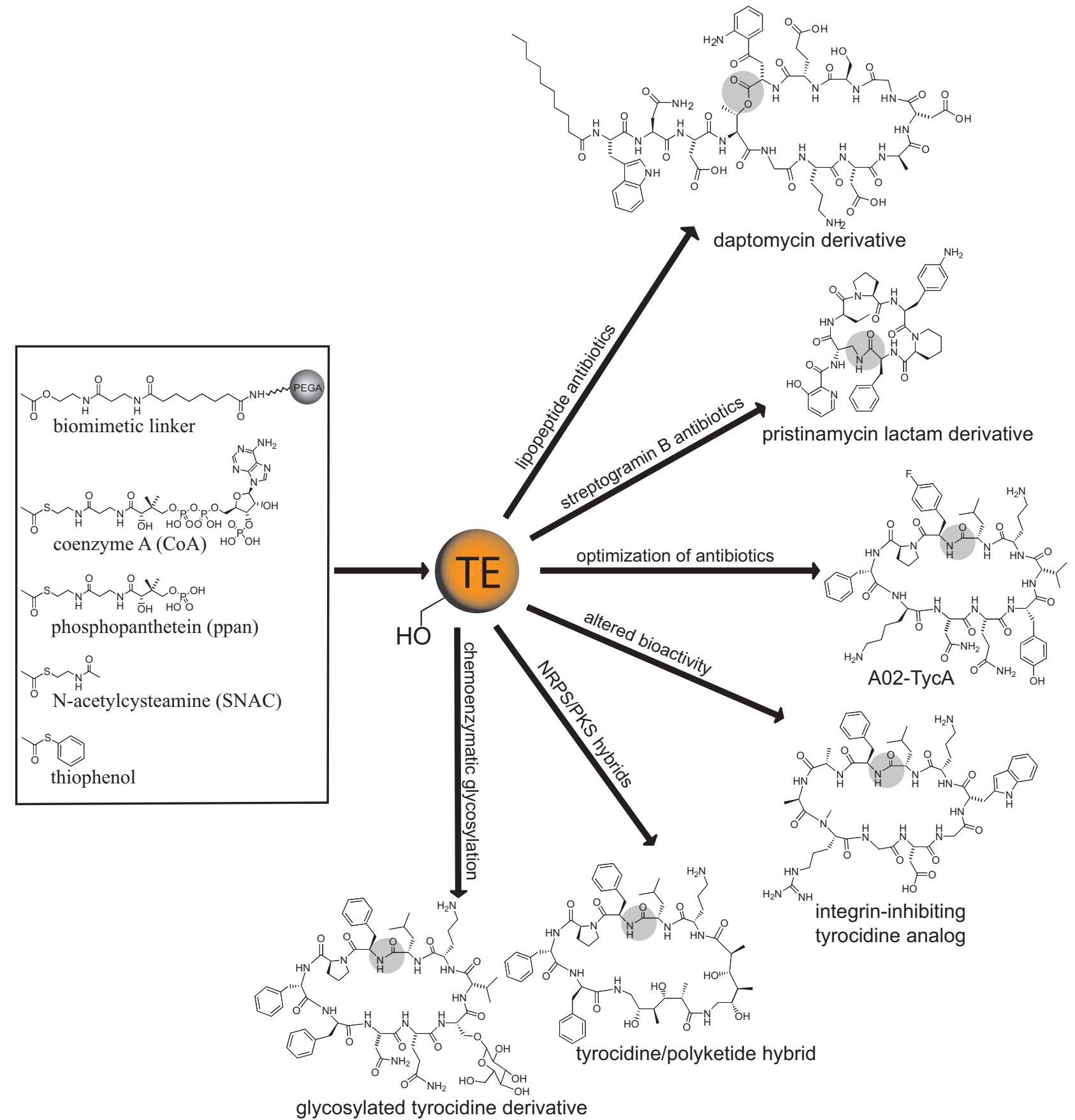

FIG. 10. Chemoenzymatic synthesis of novel bioactive compounds by excised TE-domains. Substrates can be presented to the cyclase either bound to an artificial solid support (PEGA resin) or by soluble thioester leaving group.

Interestingly, daptomycin also contains two fluorophores: Trp at the $\mathrm{N}$ terminus and nonproteinogenic Kyn at the $\mathrm{C}$ terminus. Both fluorophores show significant spectral overlap between the donor emission (Trp) and the acceptor absorption (Kyn). Remarkably, CDA TE-mediated peptide cyclization brings Trp and Kyn in sufficiently close proximity to enable efficient fluorescence resonance energy transfer (FRET), providing a tool to track TEmediated peptide cyclization in real time (43).

\section{MANIPULATION OF CARRIER PROTEINS BY POSTTRANSLATIONAL MODIFICATION}

Chemoenzymatic approaches are not limited to excised TEdomains. Recent developments indicated that carrier proteins (CPs) are ideal targets for chemoenzymatic labeling strategies with highly diverse compounds. Such CPs from NRPS, PKS, and fatty acid synthases are posttranslationally modified at a 

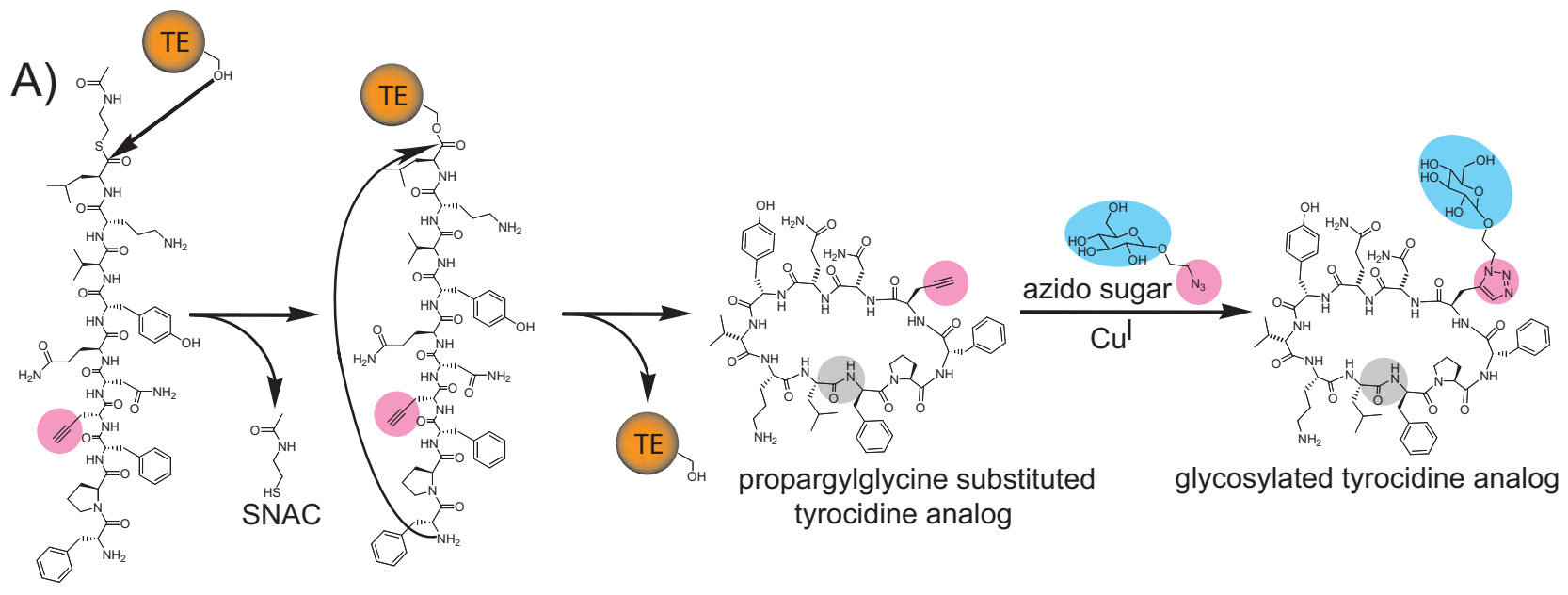

propargylglycine substituted

glycosylated tyrocidine analog

tyrocidine analog

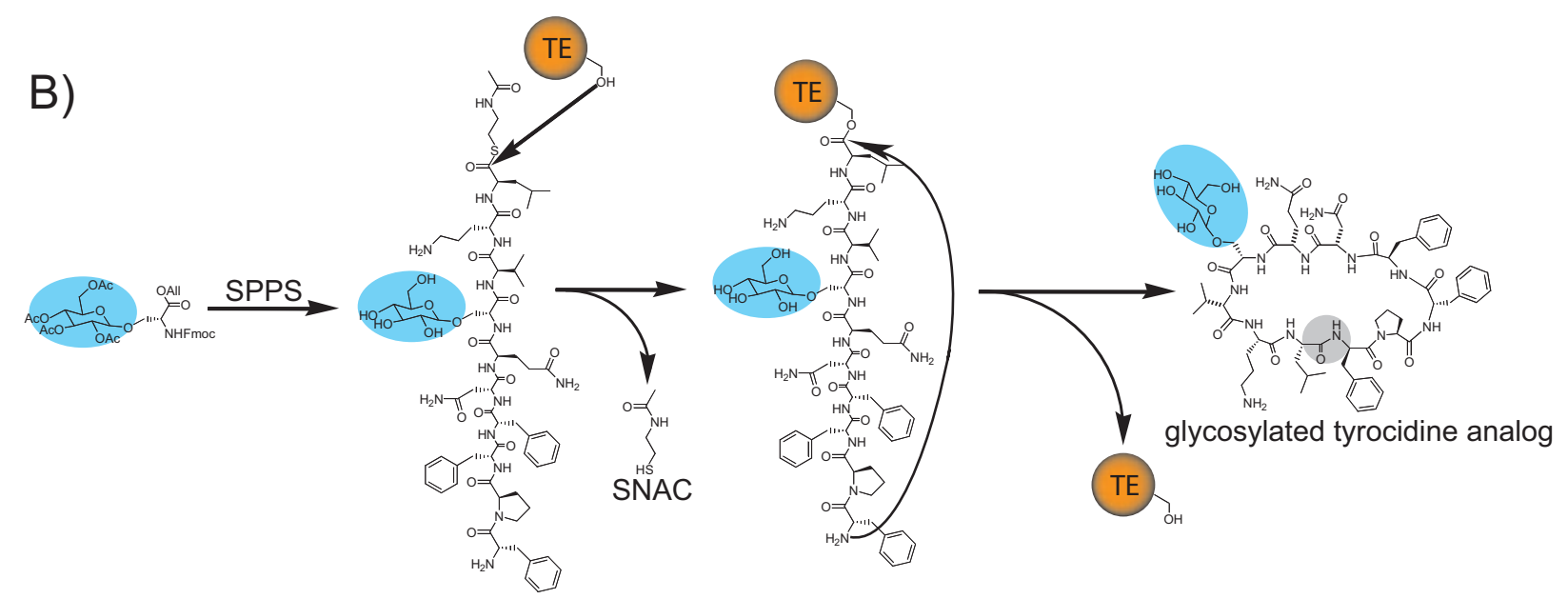

FIG. 11. Chemoenzymatic approaches to glycopeptide antibiotics. (A) A chemically synthesized propargylglycine-substituted tyrocidine SNAC substrate is cyclized by Tyc TE. The resulting alkyne-containing macrolactam is then conjugated to an azido sugar to produce a glycosylated cyclic peptide by using copper(I)-catalyzed [3 + 2] cycloaddition. (B) A glycosylated amino acid is incorporated into a linear tyrocidine SNAC thioester via SPPS. Tyc TE-catalyzed head-to-tail-cyclization then produces a glycosyl-tyrocidine analog.

conserved serine residue with a ppan moiety from CoA. This modification is catalyzed by ppan transferases, such as Sfp from B. subtilis (Fig. 12A). An interesting feature of ppan transferases is their ability to accept various functionalized CoA derivatives (Fig. 12B). This relaxed specificity has been used to tag CPs with a variety of reporter groups, such as fluorophore- and affinity-labeled $\operatorname{CoA}(71,85)$. The synthesis of such CoA conjugates can be readily achievable via Michael addition once maleimide functionalities are linked to the desired small-molecule reporter group (71).

The advantage of posttranslational modification of CPs is that this method can be selectively carried out in a complex mixture of cellular proteins. Hence, CPs can be used as peptide tags to direct the specific labeling of a target protein (Fig. 12B). Yin et al. reported the affinity labeling of target proteins that were expressed as artificial fusions to a PCP. These PCPtagged target proteins were selectively labeled with biotin in the cell lysate followed by rapid immobilization on a streptavidin surface, thereby providing a high-throughput method for protein microarray fabrication and enzymatic screening (151).
In another application, the PCP was N-terminally fused to the phage capsid protein III (152). Subsequent Sfp-catalyzed PCP modification with CoA-small-molecule conjugates enabled the display of small molecules on phage surfaces. By using this method, phagemid-encoded small molecule libraries could be screened for target binding.

In addition to phage surfaces, specific labeling of CPs with chemically diverse compounds can be achieved on cell surfaces of living cells. Recent publications provide evidence that posttranslational modification of CPs is suitable for fluorescence imaging of membrane proteins $(41,137,150)$. For instance, transferrin receptor 1 (TfR1) was fused to PCP, and the TfR1PCP fusion protein was posttranslationally labeled with fluorophore Alexa 488 by Sfp. In the presence of fluorescently labeled transferring ligand, single-cell FRET measurements provided insights into the trafficking of transferrin-TfR1-PCP complex. The observations agreed with current models for TfR1-mediated transferring uptake, thus indicating that the small size of fused PCP ( $~ 80$ aa) did not significantly alter the function of the TfR1 receptor. Similar to this approach, it was 
A)

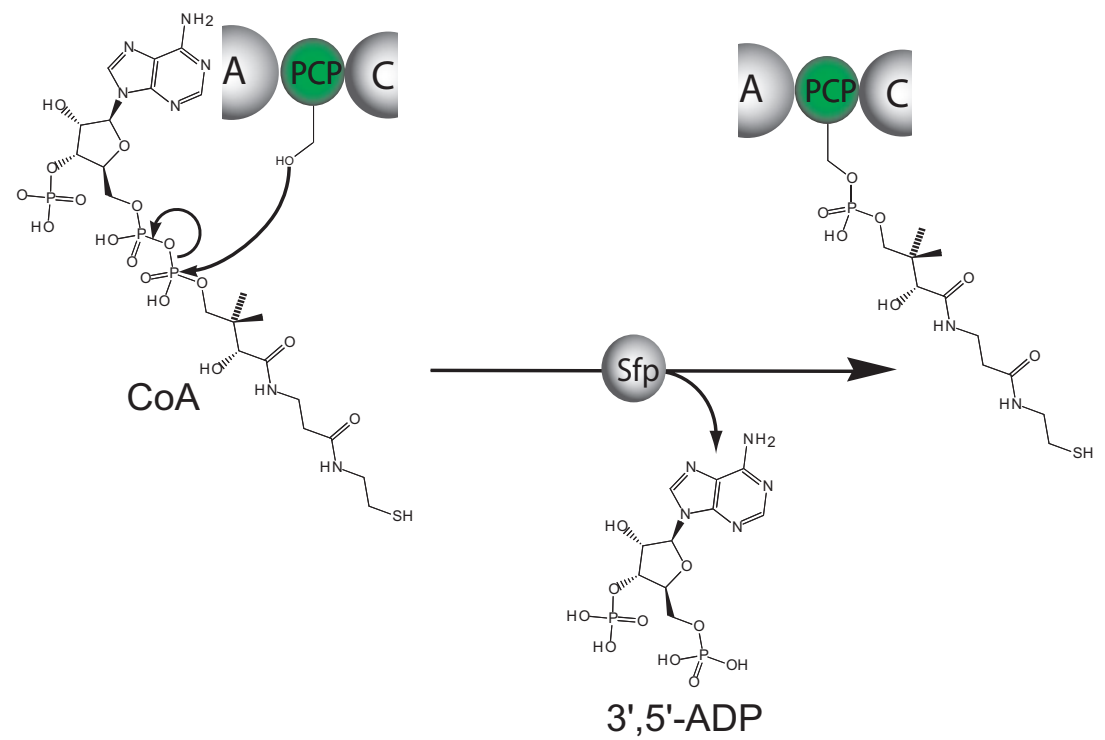

B)

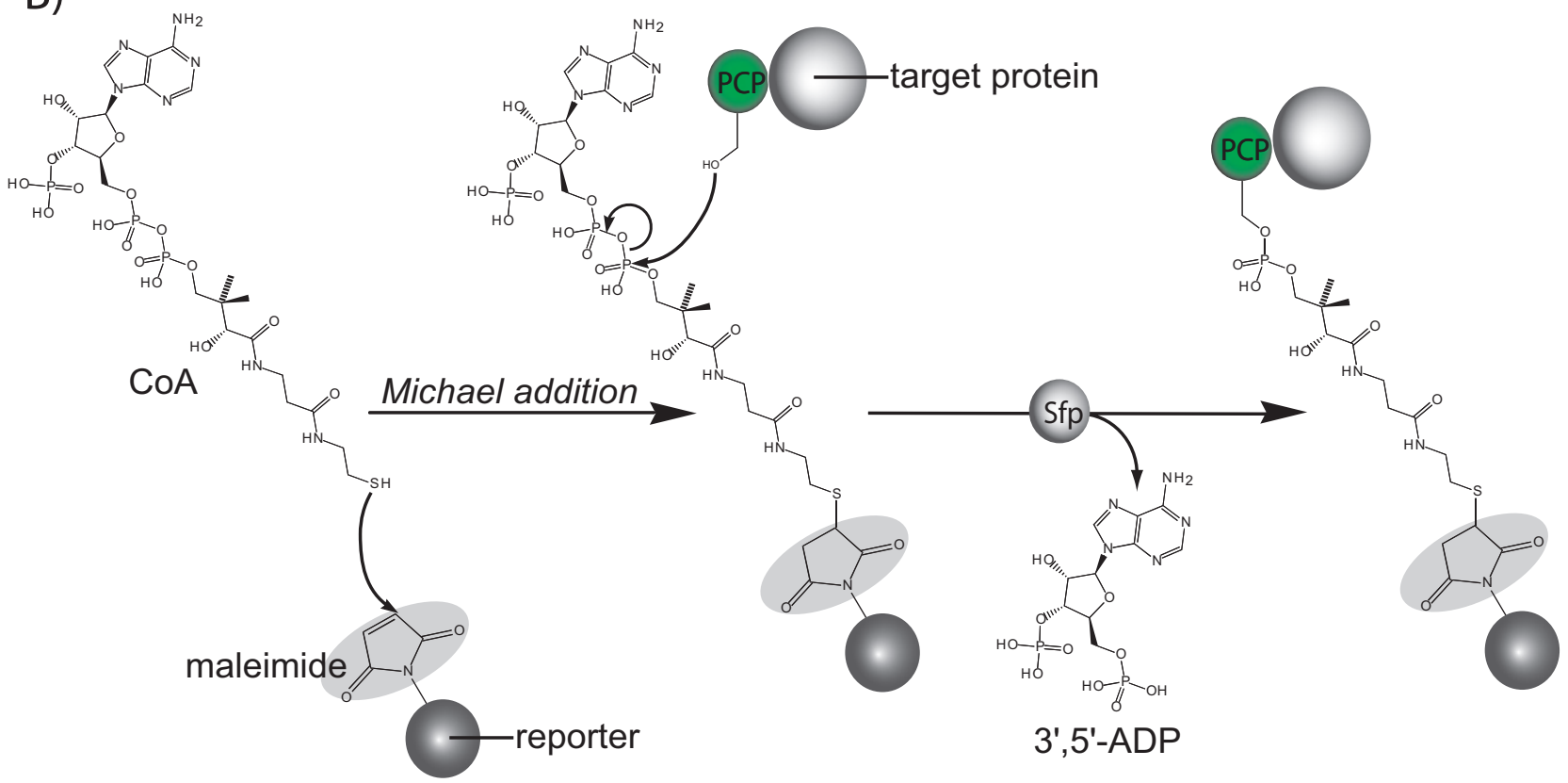

reporter $=$ fluorophore, affinity tag

FIG. 12. Posttranslational modification of PCPs. (A) Ppan transferases, such as Sfp from B. subtilis, transfer a $4^{\prime}$-phosphopantetheine residue from $\mathrm{CoA}$ to a strictly conserved serine residue of apo-PCP, creating the holo-PCP and $3^{\prime}, 5^{\prime}$-ADP. (B) CoA-reporter analogs are created through a Michael addition of the free thiol group in CoA across the double bond of reporter-linked maleimide. Subsequent Sfp-catalyzed reporter labeling of PCP fusion proteins may be used to label the target protein with small molecules, i.e., fluorophores and affinity tags.

demonstrated that a-agglutinin receptor and $\mathrm{G}$ protein-coupled receptor neurokinin-1 could be fused to the Escherichia coli ACP (41). Instead of Sfp from B. subtilis, the E. coli ppan transferase AcpS was used to achieve specific labeling of these cell surface proteins with fluorophores, affinity probes, and CdSe quantum dots.
Interestingly, AcpS has high substrate specificity and modifies only ACPs, whereas Sfp modifies both PCPs and ACPs. These enzyme properties proved useful for the multicolor imaging of two different CP fusion proteins in one sample (137). Saccharomyces cerevisiae Sag1p cell wall protein fused to ACP was first selectively modified with fluorophore-labeled $\mathrm{CoA}$ in 


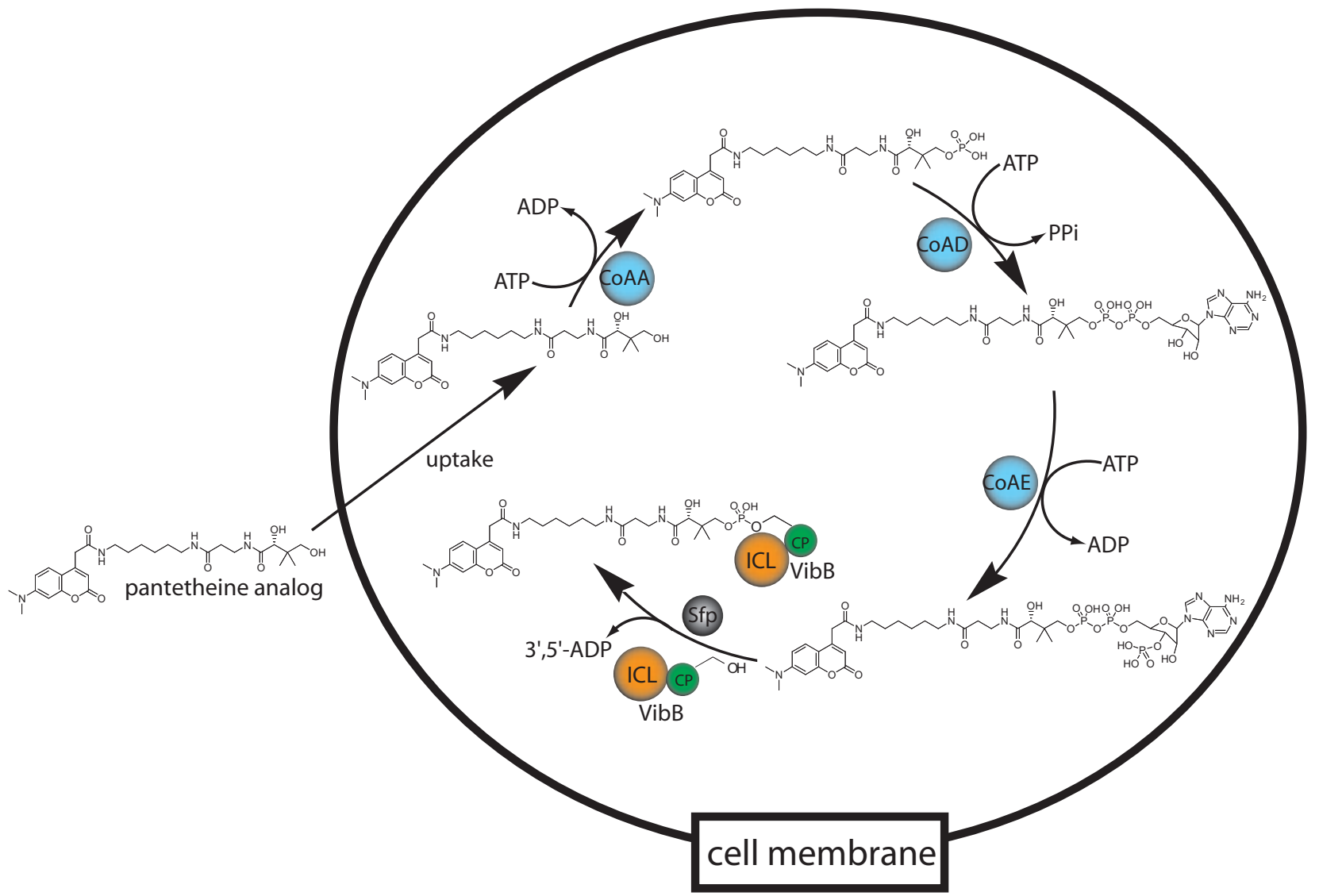

FIG. 13. In vivo tagging of carrier protein fusion VibB within E. coli. To allow cellular uptake, a cell-permeable fluorophore-labeled pantetheine analog was used. This reporter-labeled pantetheine is converted to reporter-labeled CoA by CoAA, CoAD, and CoAE in vivo. This process is followed by reaction of coexpressed Sfp to yield fluorophore-labeled VibB. CP, carrier protein; ICL, isochorismate lyase.

the presence of AcpS. Finally, Sfp catalyzed the labeling of the remaining PCP-Sag1p fusions with a different CoA-fluorophore conjugate.

The main drawback of in vivo reporter labeling of CP-tagged proteins is that the cell-impermeability of CoA derivatives limits posttranslational modification to cell surface proteins. In order to label proteins inside of cells, Clarke et al. replaced CoA-small-molecule conjugates with a cell-permeable fluorophore-labeled pantetheine analog (Fig. 13) (20). After cellular uptake in $E$. coli, this reporter-labeled pantetheine was converted to reporter-labeled $\mathrm{CoA}$ via a three-step enzymatic sequence including CoAA, CoAD, and CoAE. CoAA mediates the phosphorylation of the terminal hydroxyl group of the pantetheine analog under the consumption of ATP. Further processing by CoAD should proceed by adenylation of the generated phosphopantetheine analog to yield a dephosphoCoA derivative. CoAE-catalyzed phosphorylation of the $3^{\prime}$ hydroxyl group finally yields a CoA analog. This metabolic conversion into an active, labeled CoA analog was followed by Sfp-mediated posttranslational modification of coexpressed VibB from Vibriobacter cholerae, a natural fusion between a CP and isochorismate lyase. Labeling of VibB was confirmed by fluorescent sodium dodecyl sulfate-polyacrylamide gel electrophoresis of the cell lysate. These results demonstrated for the first time that one could rationally engineer a chemoenzymatic route to covalently label CPs in vivo via metabolic delivery of cell-permeable CoA precursors.

\section{DIVERSIFICATION AND RIGIDIFICATION OF NONRIBOSOMAL PEPTIDES MEDIATED BY TAILORING ENZYMES}

Tailoring enzymes act in the maturation of NRPS-derived products. These supplementary enzymes can carry out modifications to the peptide backbone, like $\mathrm{C}$ - and $\mathrm{N}$-methylation, oxidation, cross-linking, and halogenation, thereby enlarging the structural diversity of these natural products. Furthermore, these chemical modifications add much to the structural rigidity and stability against proteolytic digestion.

\section{C- and N-Methylation of Nonribosomal Peptides}

One striking feature of many acidic lipopeptide antibiotics is that they incorporate $\mathrm{C}$-methylated amino acids into their peptide backbones (see "DIVERSITY OF NONRIBOSOMAL PEPTIDES"). For instance, in daptomycin, CDA, and A54145, the penultimate position in the cyclopeptide is nonproteinogenic 3 mGlu. However, some A54145 and CDA variants also contain nonmethylated Glu at this position. For A54145 produced by $S$. fradiae, a temporal shift toward $3 \mathrm{mGlu}$-containing variants was observed during fermentation (13), whereas Glu-containing daptomycin/A21978C factors have not been reported to date. In the 


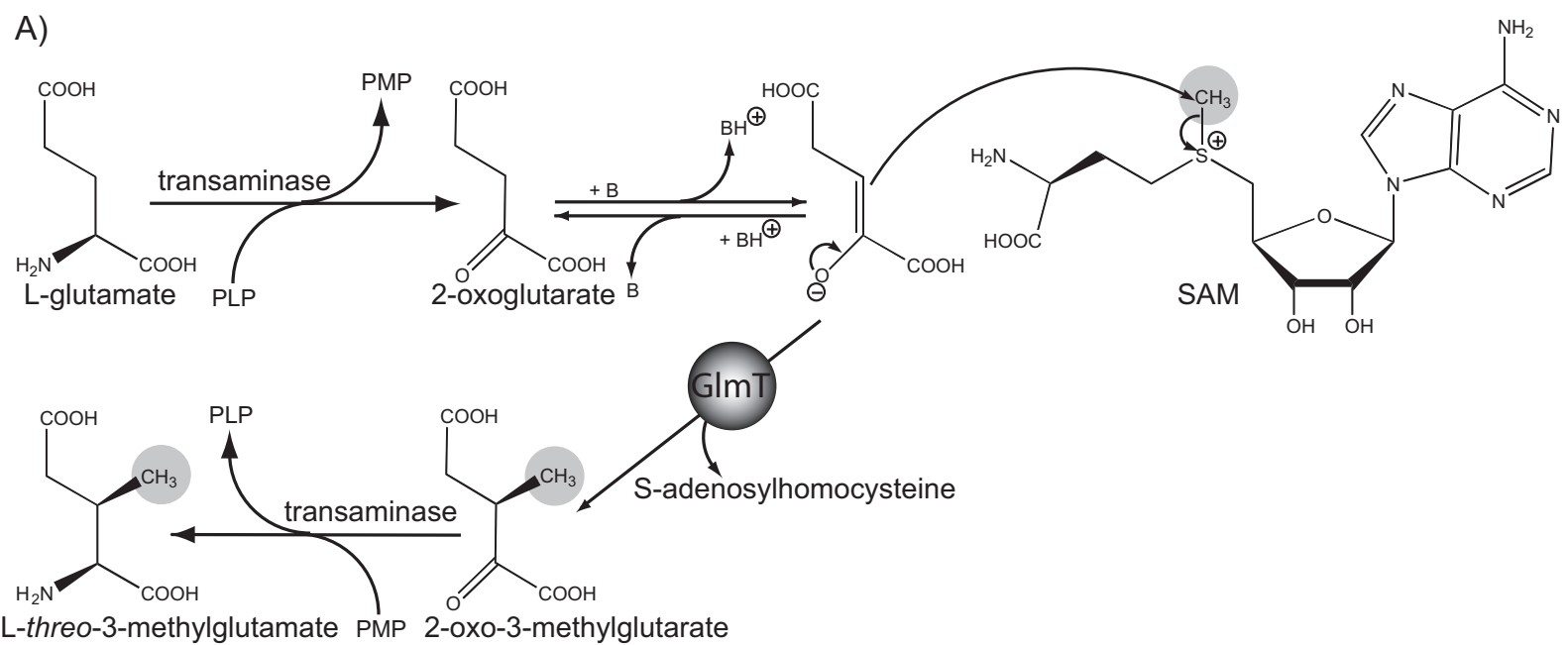

B)

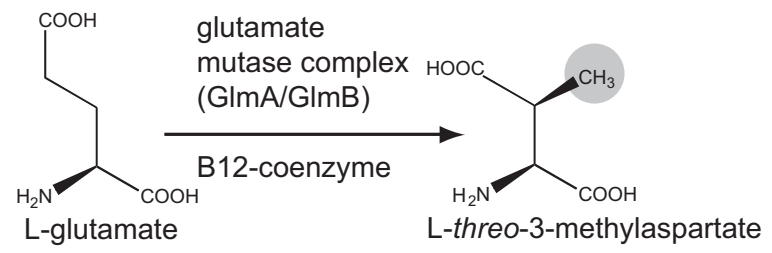

FIG. 14. C-methylation of nonribosomal peptides. (A) Proposed mechanism of C-methylation of L-glutamate in CDA. L-Glu is converted into 2-oxoglutarate in the presence of a transaminase, which uses pyridoxal phosphate (PLP) as a cofactor. After deprotonation by an as yet unknown base (B), GlmT catalyzes the stereoselective methylation of the resulting enolate by using SAM. The methylated product 2-oxo-3-methylglutarate is finally converted to L-threo-3-methylglutamate through consumption of pyridoxamine phosphate (PMP). (B) $\beta$-Methylaspartate formation in friulimicin. The conversion of L-Glu into L-threo-3-methylaspartate is mediated by a GlmA/GlmB mutase complex, which uses $B_{12}$ as a cofactor.

biosynthetic gene clusters for each of these acidic lipopeptides, the deduced translation products of the $d p t I$ gene (daptomycin) (87), the lptI gene (A54145) (86), and the $g \operatorname{lm} T$ gene (CDA) (51) are likely to have a role in $\beta$-methylation of Glu. These putative 3 mGlu methyltransferases contain three key $S$-adenosylmethionine (SAM)-dependent methyltransferase motifs (56). $\beta$-Methylation of Glu is proposed to occur prior to its activation by the cognate A-domain in the CDA biosynthetic system, because the respective A-domain differs from conventional Glu-activating domains (51). It is further speculated that Glu is converted into a more $\mathrm{C}_{\beta}-\mathrm{H}$-acidic 2-oxoglutarate by a transaminase prior to deprotonation by an as yet unknown base (Fig. 14A) (64). After methyltransferase-mediated stereoselective $\beta$-methylation, transamination of 2-oxo-3-methylglutarate leads to the formation of the final product L-threo-3mGlu. However, it cannot be ruled out to date that $\mathrm{C}$-methylation occurs directly on the nascent peptide chain or the completed peptide.

In contrast to $\beta$-methylation of glutamate in daptomycin, CDA, and A54145, a different mechanism has been proposed to be involved in the formation of analogous 3-methylaspartate found in the structures of the acidic lipopeptides amphomycin and friulimicin (48). By a reverse genetic approach, the two overlapping genes $\operatorname{glm} A$ and $\operatorname{glm} B$ were identified in the friulimicin biosynthetic gene cluster. The deduced active enzyme GlmA-GlmB probably forms a complex of two subunits. Fur- thermore, a putative cofactor $\mathrm{B}_{12}$ binding motif in GlmA suggests a glutamate mutase mechanism, which was previously described for glutamate fermentation in Clostridium sp. (16) or members of the family Enterobacteriaceae (59) (Fig. 14B). Overexpression of the resulting genes in Streptomyces lividans verified the assumed function of GlmA-GlmB as a glutamate mutase in providing 3-methylaspartate in friulimicin biosynthesis (48).

Numerous nonribosomal peptides, such as cyclosporine (Fig. 1) (135), pristinamycin (Fig. 1) (24, 80), and actinomycin (102), contain N-methylated peptide bonds. In most cases, $\mathrm{N}$-methylation is introduced by an in cis-acting $N$-methyltransferase $(\sim 420$ aa) which is inserted into the C-terminal end of the accompanying A domain. Transfer of the $S$-methyl group of SAM to the $\alpha$-amino group occurs when the respective amino acid is tethered to the ppan cofactor, whereupon amide bond formation can occur, generating an N-methylated peptide bond (102). However, in contrast to in cis-acting $N$-methyltransferases, MtfA of the chloroeremomycin biosynthetic system catalyzes in trans methyl transfer to the $\mathrm{N}$-terminal leucine of chloroeremomycin (19).

\section{Tailoring of Rigidity-Conferring Heterocyclic Elements}

In some cysteine-, serine-, and threonine-incorporating NRPS modules, the $\mathrm{C}$-domain is replaced by a cyclization domain (Cydomain) (111). In addition to peptide bond formation, the Cy- 
domain catalyzes cyclodehydration of the peptide bond to generate rigid five-membered heterocycles, such as oxazolines derived from threonine or serine, and thiazolines derived from cysteine. In many cases, Cy-domain-catalyzed heterocyclization is subsequently followed by a two-electron oxidation to form aromatic thiazoles and oxazoles. This is achieved by an oxidation domain (Ox-domain; 250 aa), which uses flavin mononucleotide as a cofactor $(30,105)$. Although Ox-domains are strictly associated with Cy-domains, they can be found in two different positions within the corresponding NRPS module: inserted into the C-terminal part of the A-domain or downstream of the PCP $(29,37,104,115)$. For instance, in epothilone synthase the Ox-domain is an integral part of the A-domain of EpoB, whereas for bleomycin, the Ox-domain is C-terminally fused to the PCP in BlmIII.

Since heterocyclic thiazoles are important determinants for bioactivity in both bleomycin and epothilone molecules, Oxdomains are interesting targets for engineering NRPSs. Entire modules containing Ox-domains were swapped into bimodular model systems, resulting in the release of unnatural oxidized dipeptide products (32). Moreover, the portability of an $\mathrm{Ox}$ domain to a heterologous NRPS assembly line was reported recently (105). Replacement of an E-domain of PchE, involved in pyochelin biosynthesis, with an Ox-domain from MtaD of the myxothiazol NRPS assembly line led to the production of a soluble flavin mononucleotide-containing chimeric module, which was assayed for oxidation activity in vitro. In fact, the chimeric module catalyzed the formation of an anticipated oxidized product, revealing the activity of the transplanted Ox-domain. Therefore, this result underscored the high portability of Ox-domains and their potential for the development of novel heterocyclic compounds.

\section{Rigidification of Peptide Scaffolds by Oxidative Cross-Linking}

During the biosynthesis of the glycopeptides vancomycin (Fig. 1) and balhimycin-these molecules share the same aglycone-three oxidative cross-linking reactions of the electron-rich phenol side chains are catalyzed by three cytochrome P450-like oxygenases (OxyA, OxyB, and OxyC) (94). These cross-links convert the acyclic, floppy heptapeptides into rigid, cup-shaped scaffolds. Gene knockout experiments with the balhimycin-producing Amycolatopsis mediterranei revealed the order of the three oxidative phenol coupling reactions that sets the rigid architecture of the heptapeptide scaffold. The first coupling reaction takes place between rings $\mathrm{C}$ and $\mathrm{D}$ and is mediated by OxyB, followed by the second cross-link formation between $\mathrm{D}$ and $\mathrm{E}$ catalyzed by OxyA. The last coupling reaction occurs between rings $\mathrm{A}$ and $\mathrm{B}$ and is catalyzed by OxyC (Fig. 15A) (10).

Further insights into the timing of oxidative cross-linking were provided by experiments with recombinant OxyB from the vancomycin NRPS (Fig. 15B) (153). The purified enzyme failed to catalyze the phenol coupling reaction of a free hexapeptide substrate. However, when the same peptide was loaded onto a PCP using the ppan transferase Sfp, incubation with OxyB resulted in $80 \%$ conversion into the desired crosslinked product. These results provide evidence that the oxidative cross-linking reaction between rings $\mathrm{C}$ and $\mathrm{D}$ takes place while the peptide intermediate is covalently attached as a thioester to a PCP of the glycopeptide assembly line. Although little is known about the cross-linking mechanism in detail, it was demonstrated that at least the catalytic action of OxyB is closely associated with the nonribosomal biosynthetic machinery.

\section{Halogenation of Electron-Rich Aromatic Rings and Unactivated Aliphatic Groups}

There are currently more than 4,000 natural products from both terrestrial and marine microorganisms, which contain carbon-chlorine, carbon-bromine, or carbon-iodine bonds (133). Most of the carbon-halogen linkages are in electron-rich aromatic or heteroaromatic ring systems, such as $3-\mathrm{Cl}-\beta-\mathrm{OH}-\mathrm{Tyr}$ of the glycopeptides vancomycin (Fig. 1), balhimycin, and chloroeremomycin (57). However, there are also natural products, which comprise unreactive aliphatic carbon atoms bearing one, two, or three chlorine atoms. For example, syringomycin E contains 4-Cl-Thr at position 9 of the peptide scaffold (46), whereas a trichlorinated-leucine-derived moiety is found in barbamide (17). Halogenation often has significant consequences for the bioactivity of these natural products. For instance, the deschloro analog of the antibiotic balhimycin was 8- to 16-fold less active against a variety of pathogenic bacteria (11).

Both aromatic and aliphatic halogenations are catalyzed by halogenases. The respective genes encoding halogenases are embedded in many NRPS and PKS biosynthetic gene clusters $(33,36,78,93,98)$. Studies with chlorination-deficient mutants of the balhimycin producer Amycolatopsis mediterranei demonstrated that a single halogenase $\mathrm{BhaA}$ is responsible for the chlorination of the two $\beta-\mathrm{OH}-\mathrm{Tyr}$ residues of the glycopeptide (97). BhaA is a representative of the $\mathrm{FADH}_{2}$-dependent halogenases, which mainly carry out halogenation of electronrich aromatic rings $(61,146)$. However, the natural substrate of BhaA and, therefore, the timing of the two halogenation reactions have remained elusive. Feeding studies with nonchlorinated $\beta-\mathrm{OH}-\mathrm{Tyr}$ indicated the biosynthesis of completely chlorinated balhimycin by a mutant strain, which is blocked in $\beta-O H-T y r$ synthesis (97). This fact clearly points to a chlorination time point later than the stage of free $\beta-\mathrm{OH}-\mathrm{Tyr}$. Moreover, mutational studies revealed that chlorination occurs at a time point prior to oxidative cross-linking and subsequent glycosylation $(94,125)$. Therefore, the timing of chlorination is proposed to occur after the $\beta-\mathrm{OH}-\mathrm{Tyr}$ residues are tethered by thioester linkages to the respective PCPs of the NRPS assembly line. This hypothesis was confirmed by investigations with the heteroaromatic 4,5-dichloropyrrole moiety of the antifungal product pyoluteorin (28). The study convincingly showed that the $\mathrm{FADH}_{2}$-dependent halogenase PltA specifically recognized the pyrrol substrate on the PCP, while free pyrrol-2carboxylate was not accepted for dichlorination by PltA. Hence, a specific interaction between the $\mathrm{FADH}_{2}$-dependent halogenase and its dedicated carrier protein must be postulated.

$\mathrm{FADH}_{2}$-dependent halogenases require molecular oxygen for activity, consistent with the formation of an FAD-OOH intermediate from $\mathrm{FADH}_{2}$ and $\mathrm{O}_{2}$, as observed for monooxygenases (82). Nucleophilic attack by a chloride ion on the proximal oxygen of FAD-OOH might then form FAD-O-Cl to provide either an electrophilic or radical chlorine species for the chlorination of electron-rich aromatic substrates $(28,147)$. 


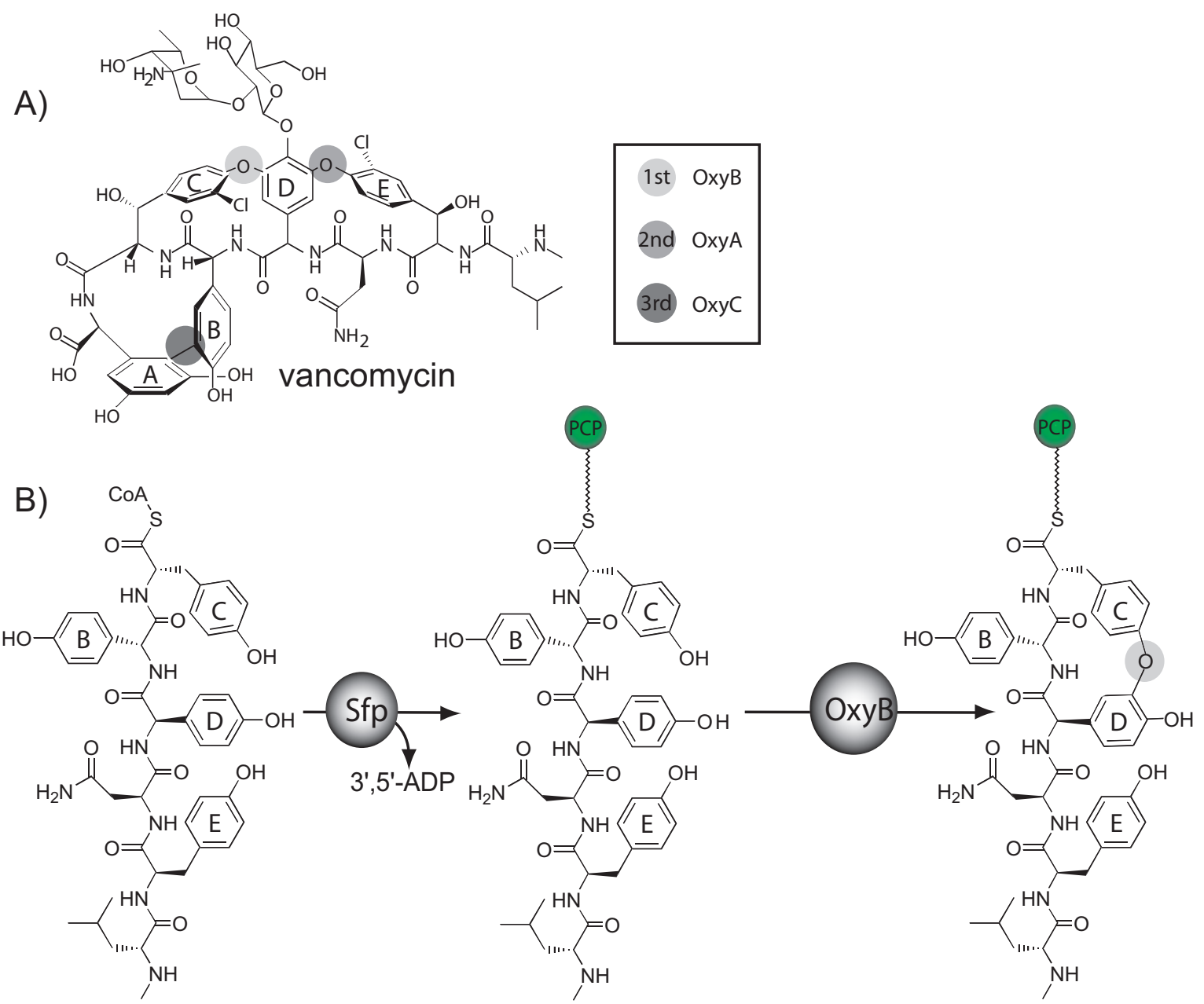

FIG. 15. Oxidative cross-linking of phenol side chains occurs only on NRPS-bound peptide chains. (A) Chemical structure of vancomycin. The order of oxidative cross-linking mediated by OxyB, OxyA, and OxyC is indicated. (B) A chemically synthesized balhimycin-like hexapeptide CoA thioester is loaded onto a PCP using Sfp. Subsequent incubation with OxyB leads to cross-linking between rings C and D.

However, aliphatic carbon atoms require a more potent chlorinating agent. Recently, Walsh and coworkers have discovered that the nonheme $\mathrm{Fe}^{2+} \alpha$-ketoglutarate- and $\mathrm{O}_{2}$-dependent halogenases $\mathrm{CmaB}$ and SyrB2 from Pseudomonas syringae catalyze the chlorination of such unactivated carbon centers (133, 134). $\mathrm{CmaB}$ encoded by the coronatine biosynthetic gene cluster chlorinates the $\gamma$-position of L-allo-isoleucine, whereas SyrB2 encoded by the syringomycin biosynthetic gene cluster catalyzes the monochlorination of the methyl group of L-Thr. It is assumed that a high-valent $\mathrm{Fe}(\mathrm{IV})=\mathrm{O}$ oxoiron species, which is a potent oxidizing and hydroxylation agent (96), is an intermediate in this chlorination pathway. After binding of a chloride ion to the $\mathrm{Fe}(\mathrm{IV})$ intermediate of the halogenase active site, a putative $\mathrm{Fe}(\mathrm{III})-\mathrm{Cl}$ species is generated, which could transfer a chlorine radical to the substrate $(133,134)$. Interestingly, both halogenases CmaB and SyrB2 specifically recognize aminoacyl thioester substrates presented on the PCPs CmaD and SyrB1. Consistent with the dichlorination of the pyrrolyl-S-PCP by $\mathrm{FADH}_{2}$-dependent halogenases during pyoluteorin biosynthesis, $\mathrm{Fe}^{2+} \alpha$-ketoglutarate- and $\mathrm{O}_{2}$-dependent halogenases do not chlorinate free sub- strates, suggesting that halogenation of aliphatic groups takes place on the modular assembly line.

\section{ENZYMES BELONGING TO THE HIGHER EUCARYOTIC NRPS-LIKE FAMILY}

Recent results demonstrate that eukaryotes have preserved an amino acid activation mechanism that until now was considered to be specific for bacterial and fungal NRPSs. Computational sequence comparisons led to the conclusion that a specificity-conferring code, similar to that described for traditional nonribosomal A-domains (see "Dissecting the Modules into Domains"), can be recognized in eukaryotic enzymes such as Ebony (27). This protein from Drosophila is a three-domain multienzyme, which is involved in histamine neurotransmitter metabolism at the photoreceptor synapse of the eye (52) (Fig. 16). It presumably functions as a fast histamine reuptake system to ensure excitation of the postsynaptic cell by disinhibition. Indeed, biochemical studies provided evidence that Ebony is capable of binding biogenic histamine as well as many 


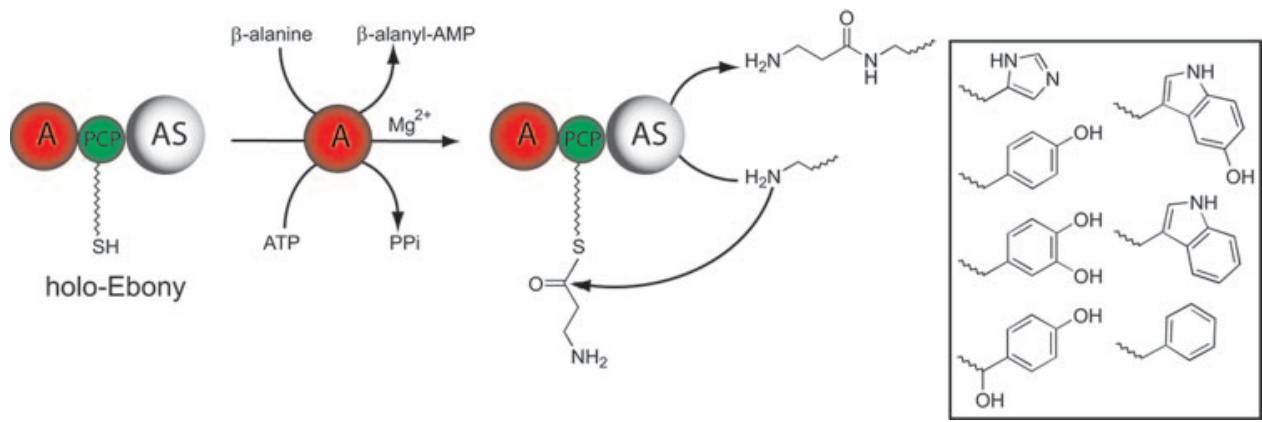

FIG. 16. Proposed mechanism for Ebony-catalyzed binding of primary amines to $\beta$-alanine. After posttranslational modification of the PCP by a dedicated ppan transferase, the A-domain of holo-Ebony specifically activates $\beta$-alanine as $\beta$-alanyl-AMP and subsequently transfers it onto the ppan cofactor. To generate the peptidoamine, the AS-domain presumably catalyzes the attack of the nucleophilic primary amine onto the electrophilic thioester of the PCP-bound $\beta$-alanine. Biochemical studies revealed that various primary amines are suitable for peptidoamine formation.

other primary amines to $\beta$-alanine (100). In vitro assays of $E$. coli-produced wild-type Ebony indicated that the respective A-domain exclusively selects $\beta$-alanine and activates it as aminoacyl adenylate. The activated $\beta$-alanine is then transferred onto the ppan cofactor of the downstream PCP in an NRPSrelated mechanism. Finally, a primary amine, such as histamine, performs a nucleophilic attack onto the thioester of the Ebony-bound $\beta$-alanine, thus leading to the release of a peptidoamine. This condensation step might be catalyzed by a C-terminal amine-selecting domain (AS-domain; 230 aa), which does not share homology with any known NRPSs. Furthermore, condensation assays suggested a broad substrate tolerance of this domain for various primary amines. A combination of an ethylamino or hydroxyethylamino group with an aromatic ring system was sufficient for rapid peptidoamine formation.

Similar to the Ebony three-domain NRPS, a multifunctional enzyme from mouse, U26, comprises an A-domain, a PCP, and a pyrroloquinoline quinone-dependent dehydrogenase domain (58). The latter domain has been proposed to catabolize lysine, which yields $\alpha$-aminoadipic acid. In conclusion, these recent developments indicate that NRPS architecture has been preserved throughout evolution to higher eukaryotes. However, multimodular NRPS systems have not yet been detected, even though dipeptides such as $\beta$-alanyl-histidine have been shown to exist in vertebrates (38).

\section{REPROGRAMMING OF NRPS ASSEMBLY LINES}

Chemoenzymatic approaches were developed to reprogram natural peptide sequences by the combined action of chemical peptide synthesis and subsequent enzyme catalysis (see "Chemoenzymatic Approaches toward Novel Cyclopeptides”). By using the combinatorial method described above (69), large libraries of macrocyclic peptides can be created with both natural and unnatural amino acid building blocks, which can subsequently be screened for novel or improved bioactivity. Once a target analog is unveiled by this method, the modular organization of NRPSs makes it possible to consider reprogramming of the biosynthetic machinery to generate the target analog by fermentation. Several strategies to rationally rede- sign NRPS templates are conceivable and will be introduced in this section.

Pioneering work was performed by Stachelhaus et al., who reported the genetic engineering of the terminal module of the surfactin synthetase, which incorporates $\mathrm{Leu}_{7}$ into the final product (119). To alter the substrate specificity of this module in vivo, the $\mathrm{A}_{7}$-domain and the adjacent $\mathrm{PCP}_{7}$ were exchanged by bacterial and fungal A-PCP didomains with various amino acid specificities. Despite the production of the predicted surfactin derivatives, the productivity of the engineered synthetases was dramatically reduced, which could be explained by the high selectivity of C-domains in the acceptor site for cognate side chains (7). However, further attempts to obtain other surfactin variants by domain swapping were unsuccessful (103). This can most probably be ascribed to improper artificial fusion of domains, thus indicating that a more precise definition of the domain borders was necessary. Further biochemical studies, detailed sequence analysis, and structural information led to the identification of linker regions between independently folding NRPS domains $(22,60,90,141)$. These linker regions are about 15 amino acids in length and usually show only little or no sequence conservation. Their suitability for artificial module fusions was first confirmed by in vitro studies on the tyrocidine NRPS. Dimodular hybrid enzymes were generated by fusion of Pro-activating module 2 with Ornactivating module 9 or Leu-activating module 10 (90). Furthermore, a TE-domain was fused to the terminal modules to ensure product release. Incubation of the engineered dimodules with the D-Phe-activating module 1 then yielded the predicted tripeptides D-Phe-Pro-Orn and D-Phe-ProLeu. Moreover, precise linker surgery significantly improved NRPS engineering efforts in vivo. To this end, successful deletion of the entire Leu-activating module 2 of the surfactin synthetase caused the secretion of the predicted lipopeptidolactone analog with a decreased ring size (89). The yield of this $\Delta 2$-surfactin variant was around $10 \%$ of native surfactin A (Fig. 1) produced by the wild-type producer, which represents a major improvement to initial in vivo studies of NRPS engineering (119).

The aforementioned NRPS reprogramming efforts have fo- 

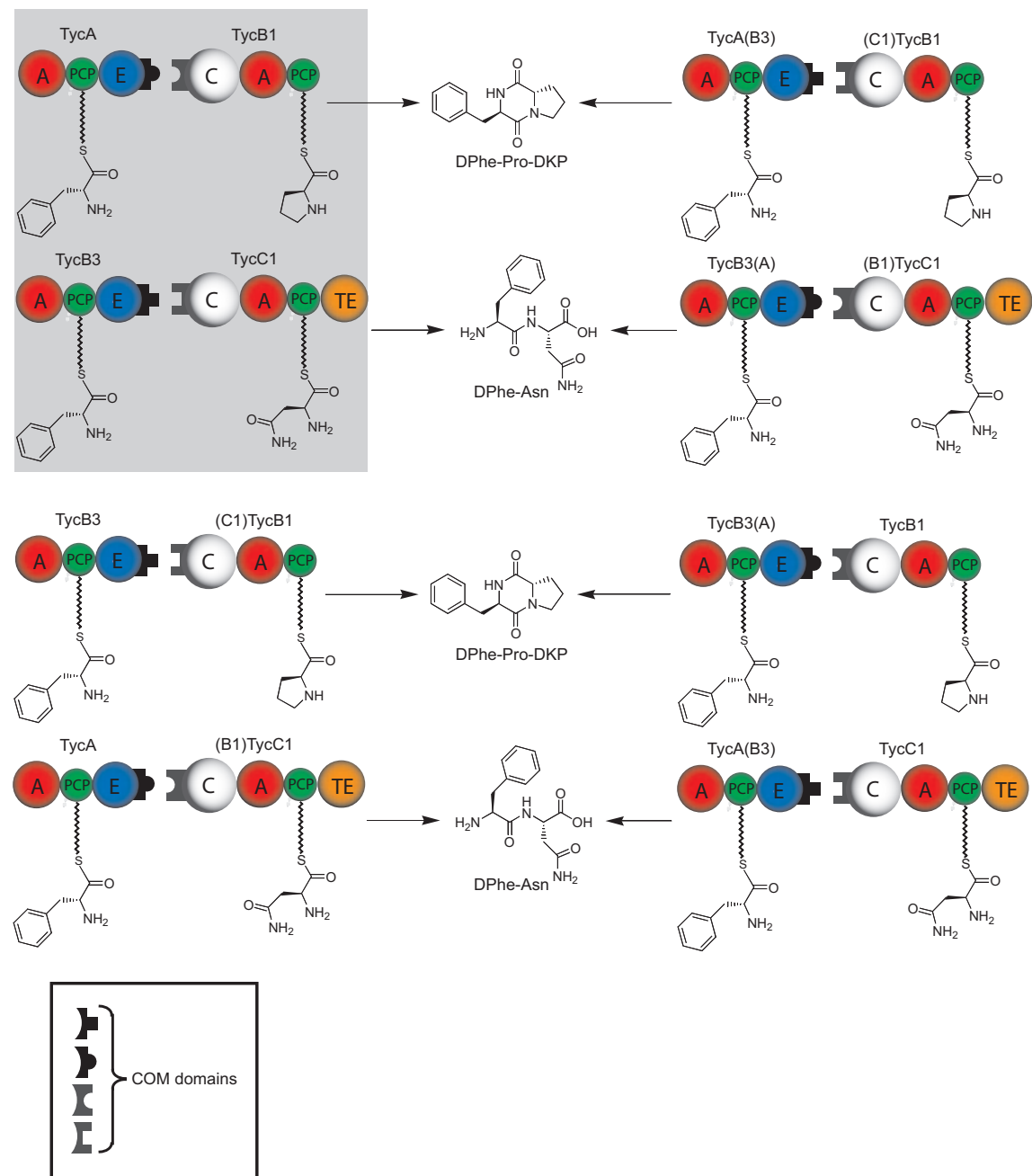

FIG. 17. COM domain swapping (origin of the proposed COM domains are in parentheses). Native in trans communication between NRPS modules of tyrocidine synthetase is indicated by shading. Productive interaction between these partner modules can also be enforced by the presence of matching COM domains derived from different modules $[\mathrm{TycA}(\mathrm{B} 3) /(\mathrm{C} 1) \mathrm{TycB} 1$ and TycB3(A)/(B1)TycC1]. Likewise, cross talk can be induced between nonpartner NRPSs [TycB3/(C1)TycB1, TycA/(B1)TycC1, TycB3(A)/TycB1, and TycA(B3)/TycC1].

cused on the interaction between modules within NRPS subunits in cis. To expand the biosynthetic utility of NRPSs, recent research focused on interpolypeptide interactions between modules of partner subunits in trans. The predictable manipulation of these interactions would provide a great combinatorial potential. Interestingly, protein-protein communication in trans is predominantly controlled by the interplay of matching pairs of short COM domains (see "BIOSYNTHETIC LOGIC OF NONRIBOSOMAL PEPTIDE SYNTHETASES”). Recent studies of the tyrocidine NRPS subunits TycA, TycB, and TycC convincingly showed that productive interactions between nonpartner NRPS subunits can be enforced by the presence of matching pairs of COM domains (Fig. 17) (47). Specifically, COM domain swapping enabled cross talk between the D-Phe-activating donor module TycA and the nonpartner Asn-activating acceptor module TycC1 as well as between the D-Phe-activating donor module TycB3 and the Pro-activating acceptor module TycB1. Formation of the expected dipeptides D-Phe-Asn and D-Phe-Pro-diketopiperazine was verified by mass spectrometry. Remarkably, communication between partner mod- ules was also achieved by COM domain pairs derived from different matching modules, i.e., TycA(B3)/(C1)TycB1 and TycB3(A)/(B1)TycC1 (Fig. 17). Moreover, successful cross talk between TycA and the Leu-activating termination module SrfAC of surfactin synthetase indicated that COM domains even mediate protein-protein communication between different biosynthetic systems. In the future, it will be an important issue to clarify if COM domain swapping is also a suitable tool for the production of novel peptide products in vivo.

The engineering of intra- and interpolypeptide interactions between whole modules represents a rather drastic intervention in NRPS biosynthesis. Genetic manipulation of the nonribosomal code (see "Dissecting the Modules into Domains") by site-directed mutagenesis of the specificity-conferring residues of A-domains, on the other hand, is a rather small alteration. Studies of CDA and surfactin synthetase have shown that this approach is suitable to produce novel peptide products in vivo $(35,132)$. For example, the substrate specificity of the Asp-activating module 7 of CDA was rationally altered by changing two residues within the corresponding A-domain 
(132). Only two point mutations resulted in a CDA2a analog (Fig. 2), which contained Asn instead of Asp at position 7. However, fermentation yields of the $\mathrm{Asn}_{7}$-containing cyclopeptide were reduced, and large amounts of a linear hexapeptide by-product were isolated. Hence, this method does not overcome the limitation imposed by the specificity of the acceptor site of the C-domain, which is located upstream of the manipulated A-domain. Moreover, the substrate specificity of the C-terminal TE-domain could cause additional problems.

Interestingly, engineered biosynthesis of non-ribosomally produced peptides can be performed in vivo without genetic manipulation of the NRPS subunits. Instead, engineering of these natural products is achieved by deleting a gene required for the biosynthesis of an unusual amino acid. Feeding synthetic analogs of this unusual amino acid then results in new peptide analogs by precursor-directed biosynthesis. Using this so-called mutasynthesis approach, it was possible to engineer the biosynthesis of balhimycin (142) and CDA (51). In the latter case, a mutant that is blocked in HPG biosynthesis and is therefore unable to produce CDA was generated. By feeding the mutant cells a series of synthetic analogs of HPG, novel CDA peptides containing 4-fluorophenylglycine or phenylglycine in place of HPG were synthesized. However, substitutions are limited to derivatives of HPG which contain para-substituents that are no larger than the hydroxyl group (51). Presumably, the substrate specificity of the HPG-activating A-domain imposes severe restrictions on the size of this para-substituent. Furthermore, in contrast to the approaches described above, mutasynthesis is limited to substitutions of nonproteinogenic amino acids, which originate from secondary metabolism. Knowledge of the genes involved in the synthesis of these unusual amino acids is also crucial for targeted mutation and subsequent precursor directed biosynthesis.

\section{CLOSING REMARKS}

Non-ribosomally produced peptides exhibit antibacterial, antiviral, immunosuppressive, and antitumor properties. This broad spectrum of biological activities is reflected in the vast structural diversity found in these natural products such as D-configured residues, oxidation, methylation, halogenation, lipidation, heterocyclization, and macrocyclization. The latter structural feature can be chemoenzymatically generated by excised TE-domains. Future research will show if these enzymes are well-suited for the identification of drug leads via combinatorial synthesis of cyclopeptides. Furthermore, alteration of the substrate specificity of TE-domains by directed protein evolution will increase the utility of these macrocyclization catalysts. In contrast to the case with TE-domains, little is known about the chemoenzymatic potential of tailoring enzymes, which significantly contribute to the structural diversity and rigidity of nonribosomal peptides. It remains to be seen whether these enzymes exhibit a high tolerance in vitro for their dedicated chemical transformations. In addition to singledomain catalysis, reprogramming of entire NRPS assembly lines and mutasynthesis have also proven valuable for the generation of novel peptide products.

\section{ACKNOWLEDGMENTS}

We would like to thank all members of the Marahiel group for helpful discussions as well as Christoph Mahlert, Florian Kopp, and Stephan A. Sieber for carefully reading the manuscript.

This work was supported by grants from the Deutsche Forschungs-

gemeinschaft (to M.A.M.), the European Union (to M.A.M.), and the Fonds der Chemischen Industrie (to J.G. and M.A.M.).

\section{REFERENCES}

1. Balibar, C. J., F. H. Vaillancourt, and C. T. Walsh. 2005. Generation of D-amino acid residues in assembly of arthrofactin by dual condensation/ epimerization domains. Chem. Biol. 12:1189-1200.

2. Ball, L. J., C. M. Goult, J. A. Donarski, J. Micklefield, and V. Ramesh. 2004. NMR structure determination and calcium binding effects of lipopeptide antibiotic daptomycin. Org. Biomol. Chem. 2:1872-1878.

3. Baltz, R. H., P. Brian, V. Miao, and S. K. Wrigley. 2005. Combinatorial biosynthesis of lipopeptide antibiotics in Streptomyces roseosporus. J. Ind. Microbiol. Biotechnol. 2005:1-9. [Online.]

4. Barna, J. C., and D. H. Williams. 1984. The structure and mode of action of glycopeptide antibiotics of the vancomycin group. Annu. Rev. Microbiol. 38:339-357.

5. Beck, Z. Q., C. C. Aldrich, N. A. Magarvey, G. I. Georg, and D. H. Sherman. 2005. Chemoenzymatic synthesis of cryptophycin/arenastatin natural products. Biochemistry 44:13457-13466.

6. Becker, J. E., R. E. Moore, and B. S. Moore. 2004. Cloning, sequencing, and biochemical characterization of the nostocyclopeptide biosynthetic gene cluster: molecular basis for imine macrocyclization. Gene 325:35-42.

7. Belshaw, P. J., C. T. Walsh, and T. Stachelhaus. 1999. Aminoacyl-CoAs as probes of condensation domain selectivity in nonribosomal peptide synthesis. Science 284:486-489.

8. Berdy, J. 2005. Bioactive microbial metabolites. J. Antibiot. (Tokyo) 58:1-26.

9. Bergendahl, V., U. Linne, and M. A. Marahiel. 2002. Mutational analysis of the C-domain in nonribosomal peptide synthesis. Eur. J. Biochem. 269:620629.

10. Bischoff, D., S. Pelzer, B. Bister, G. J. Nicholson, S. Stockert, M. Schirle, W. Wohlleben, G. Jung, and R. D. Sussmuth. 2001. The biosynthesis of vancomycin-type glycopeptide antibiotics-the order of the cyclization steps. Angew. Chem. Int. Ed. Engl. 40:4688-4691.

11. Bister, B., D. Bischoff, G. J. Nicholson, S. Stockert, J. Wink, C. Brunati, S. Donadio, S. Pelzer, W. Wohlleben, and R. D. Sussmuth. 2003. Bromobalhimycin and chlorobromobalhimycins-illuminating the potential of halogenases in glycopeptide antibiotic biosyntheses. Chembiochem 4:658-662.

12. Boddy, C. N., T. L. Schneider, K. Hotta, C. T. Walsh, and C. Khosla. 2003. Epothilone $\mathrm{C}$ macrolactonization and hydrolysis are catalyzed by the isolated thioesterase domain of epothilone polyketide synthase. J. Am. Chem. Soc. 125:3428-3429.

13. Boeck, L. D., H. R. Papiska, R. W. Wetzel, J. S. Mynderse, D. S. Fukuda, F. P. Mertz, and D. M. Berry. 1990. A54145, a new lipopeptide antibiotic complex: discovery, taxonomy, fermentation and HPLC. J Antibiot. (Tokyo) 43:587-593

14. Broadhurst, R. W., D. Nietlispach, M. P. Wheatcroft, P. F. Leadlay, and K. J. Weissman. 2003. The structure of docking domains in modular polyketide synthases. Chem. Biol. 10:723-731.

15. Bruner, S. D., T. Weber, R. M. Kohli, D. Schwarzer, M. A. Marahiel, C. T. Walsh, and M. T. Stubbs. 2002. Structural basis for the cyclization of the lipopeptide antibiotic surfactin by the thioesterase domain SrfTE. Structure (Cambridge) 10:301-310.

16. Buckel, W. 2001. Unusual enzymes involved in five pathways of glutamate fermentation. Appl. Microbiol. Biotechnol. 57:263-273.

17. Chang, Z., P. Flatt, W. H. Gerwick, V. A. Nguyen, C. L. Willis, and D. H. Sherman. 2002. The barbamide biosynthetic gene cluster: a novel marine cyanobacterial system of mixed polyketide synthase (PKS)-non-ribosomal peptide synthetase (NRPS) origin involving an unusual trichloroleucyl starter unit. Gene 296:235-247.

18. Chatterjee, C., M. Paul, L. Xie, and W. A. van der Donk. 2005. Biosynthesis and mode of action of lantibiotics. Chem. Rev. 105:633-684.

19. Chen, H., S. O'Connor, D. E. Cane, and C. T. Walsh. 2001. Epothilone biosynthesis: assembly of the methylthiazolylcarboxy starter unit on the EpoB subunit. Chem. Biol. 8:899-912.

20. Clarke, K. M., A. C. Mercer, J. J. La Clair, and M. D. Burkart. 2005. In vivo reporter labeling of proteins via metabolic delivery of coenzyme A analogues. J. Am. Chem. Soc. 127:11234-11235.

21. Clugston, S. L., S. A. Sieber, M. A. Marahiel, and C. T. Walsh. 2003. Chirality of peptide bond-forming condensation domains in nonribosomal peptide synthetases: the C5 domain of tyrocidine synthetase is a $(\mathrm{D}) \mathrm{C}(\mathrm{L})$ catalyst. Biochemistry 42:12095-12104.

22. Conti, E., T. Stachelhaus, M. A. Marahiel, and P. Brick. 1997. Structural basis for the activation of phenylalanine in the non-ribosomal biosynthesis of gramicidin S. EMBO J. 16:4174-4183. 
23. Davies, J. S. 2003. The cyclization of peptides and depsipeptides. J. Pept. Sci. 9:471-501.

24. de Crecy-Lagard, V., V. Blanc, P. Gil, L. Naudin, S. Lorenzon, A. Famechon, N. Bamas-Jacques, J. Crouzet, and D. Thibaut. 1997. Pristinamycin I biosynthesis in Streptomyces pristinaespiralis: molecular characterization of the first two structural peptide synthetase genes. J. Bacteriol. 179:705-713.

25. Debono, M., B. J. Abbott, R. M. Molloy, D. S. Fukuda, A. H. Hunt, V. M. Daupert, F. T. Counter, J. L. Ott, C. B. Carrell, L. C. Howard, et al. 1988 Enzymatic and chemical modifications of lipopeptide antibiotic A21978C: the synthesis and evaluation of daptomycin (LY146032). J. Antibiot. (Tokyo) 41:1093-1105

26. Dieckmann, R., Y. O. Lee, H. van Liempt, H. von Dohren, and $\mathbf{H}$. Kleinkauf. 1995. Expression of an active adenylate-forming domain of peptide synthetases corresponding to acyl-CoA-synthetases. FEBS Lett. 357:212-216

27. Di Vincenzo, L., I. Grgurina, and S. Pascarella. 2005. In silico analysis of the adenylation domains of the freestanding enzymes belonging to the eucaryotic nonribosomal peptide synthetase-like family. FEBS J. 272:929-941.

28. Dorrestein, P. C., E. Yeh, S. Garneau-Tsodikova, N. L. Kelleher, and C. T. Walsh. 2005. Dichlorination of a pyrrolyl-S-carrier protein by FADH2 dependent halogenase PltA during pyoluteorin biosynthesis. Proc. Natl. Acad. Sci. USA 102:13843-13848.

29. Du, L., M. Chen, C. Sanchez, and B. Shen. 2000. An oxidation domain in the BlmIII nonribosomal peptide synthetase propably catalyzing thiazole formation in the biosynthesis of the anti-tumor drug bleomycin in Streptomyces verticillus ATCC15003. FEMS Microbiol. Lett. 189:171-175.

30. Du, L., C. Sanchez, M. Chen, D. J. Edwards, and B. Shen. 2000. The biosynthetic gene cluster for the antitumor drug bleomycin from Streptomyces verticillus ATCC15003 supporting functional interactions between nonribosomal peptide synthetases and a polyketide synthase. Chem. Biol 7:623-642.

31. Du, L., C. Sanchez, and B. Shen. 2001. Hybrid peptide-polyketide natural products: biosynthesis and prospects toward engineering novel molecules. Metab. Eng. 3:78-95.

32. Duerfahrt, T., K. Eppelmann, R. Muller, and M. A. Marahiel. 2004. Rational design of a bimodular model system for the investigation of heterocyclization in nonribosomal peptide biosynthesis. Chem. Biol. 11:261-271.

33. Edwards, D. J., B. L. Marquez, L. M. Nogle, K. McPhail, D. E. Goeger, M. A. Roberts, and W. H. Gerwick. 2004. Structure and biosynthesis of the jamaicamides, new mixed polyketide-peptide neurotoxins from the marine cyanobacterium Lyngbya majuscula. Chem. Biol. 11:817-833.

34. Ehmann, D. E., C. A. Shaw-Reid, H. C. Losey, and C. T. Walsh. 2000. The EntF and EntE adenylation domains of Escherichia coli enterobactin synthetase: sequestration and selectivity in acyl-AMP transfers to thiolation domain cosubstrates. Proc. Natl. Acad. Sci. USA 97:2509-2514.

35. Eppelmann, K., T. Stachelhaus, and M. A. Marahiel. 2002. Exploitation of the selectivity-conferring code of nonribosomal peptide synthetases for the rational design of novel peptide antibiotics. Biochemistry 41:9718-9726.

36. Eustaquio, A. S., B. Gust, T. Luft, S. M. Li, K. F. Chater, and L. Heide. 2003. Clorobiocin biosynthesis in Streptomyces: identification of the halogenase and generation of structural analogs. Chem. Biol. 10:279-288.

37. Finking, R., and M. A. Marahiel. 2004. Biosynthesis of nonribosomal peptides. Annu. Rev. Microbiol. 58:453-488.

38. Flancbaum, L., D. N. Brotman, J. C. Fitzpatrick, T. Van Es, E. Kasziba, and H. Fisher. 1990. Existence of carcinine, a histamine-related compound, in mammalian tissues. Life Sci. 47:1587-1593.

39. Fukuda, D. S., R. H. Du Bus, P. J. Baker, D. M. Berry, and J. S. Mynderse. 1990. A54145, a new lipopeptide antibiotic complex: isolation and characterization. J. Antibiot. (Tokyo) 43:594-600.

40. Garbe, D., S. A. Sieber, N. G. Bandur, U. Koert, and M. A. Marahiel. 2004. Enzymatic cyclisation of peptidomimetics with incorporated (E)-alkene dipeptide isosteres. Chembiochem 5:1000-1003.

41. George, N., H. Pick, H. Vogel, N. Johnsson, and K. Johnsson. 2004. Specific labeling of cell surface proteins with chemically diverse compounds. J. Am. Chem. Soc. 126:8896-8897.

42. Golakoti, T., W. Y. Yoshida, S. Chaganty, and R. E. Moore. 2001. Isolation and structure determination of nostocyclopeptides A1 and A2 from the terrestrial cyanobacterium Nostoc sp. ATCC53789. J. Nat. Prod. 64:54-59.

43. Grünewald, J., F. Kopp, C. Mahlert, U. Linne, S. A. Sieber, and M. A. Marahiel. 2005. Fluorescence resonance energy transfer as a probe of peptide cyclization catalyzed by nonribosomal thioesterase domains. Chem. Biol. 12:873-881.

44. Grünewald, J., S. A. Sieber, C. Mahlert, U. Linne, and M. A. Marahiel. 2004. Synthesis and derivatization of daptomycin: a chemoenzymatic route to acidic lipopeptide antibiotics. J. Am. Chem. Soc. 126:17025-17031.

45. Grünewald, J., S. A. Sieber, and M. A. Marahiel. 2004. Chemo- and regioselective peptide cyclization triggered by the $\mathrm{N}$-terminal fatty acid chain length: the recombinant cyclase of the calcium-dependent antibiotic from Streptomyces coelicolor. Biochemistry 43:2915-2925.

46. Guenzi, E., G. Galli, I. Grgurina, D. C. Gross, and G. Grandi. 1998 Characterization of the syringomycin synthetase gene cluster. A link be- tween prokaryotic and eukaryotic peptide synthetases. J. Biol. Chem. 273: 32857-32863.

47. Hahn, M., and T. Stachelhaus. 2004 Selective interaction between nonribosomal peptide synthetases is facilitated by short communication-mediating domains. Proc. Natl. Acad. Sci. USA 101:15585-15590.

48. Heinzelmann, E., S. Berger, O. Puk, B. Reichenstein, W. Wohlleben, and D. Schwartz. 2003. A glutamate mutase is involved in the biosynthesis of the lipopeptide antibiotic friulimicin in Actinoplanes friuliensis. Antimicrob. Agents Chemother. 47:447-457.

49. Hill, J., J. Siedlecki, I. Parr, M. Morytko, X. Yu, Y. Zhang, J. Silverman, N. Controneo, V. Laganas, T. Li, J. J. Lai, D. Keith, G. Shimer, and J. Finn. 2003. Synthesis and biological activity of $\mathrm{N}$-acylated ornithine analogues of daptomycin. Bioorg. Med. Chem. Lett. 13:4187-4191.

50. Hoffmann, K., E. Schneider-Scherzer, H. Kleinkauf, and R. Zocher. 1994 Purification and characterization of eucaryotic alanine racemase acting as key enzyme in cyclosporin biosynthesis. J. Biol. Chem. 269:12710-12714.

51. Hojati, Z., C. Milne, B. Harvey, L. Gordon, M. Borg, F. Flett, B. Wilkinson, P. J. Sidebottom, B. A. Rudd, M. A. Hayes, C. P. Smith, and J. Micklefield. 2002. Structure, biosynthetic origin, and engineered biosynthesis of calcium-dependent antibiotics from Streptomyces coelicolor. Chem. Biol. 9:1175-1187.

52. Hovemann, B. T., R. P. Ryseck, U. Walldorf, K. F. Stortkuhl, I. D. Dietzel, and E. Dessen. 1998. The Drosophila ebony gene is closely related to microbial peptide synthetases and shows specific cuticle and nervous system expression. Gene 221:1-9.

53. Jackowski, S. 1996. Biosynthesis of pantothenic acid and coenzyme A, p 687-694. In F. C. Neidhart, R. Curtiss III, J. L. Ingraham, E. C. C. Lin, K. B. Low, B. Magasanik, W. S. Reznikoff, M. Riley, M. Schaechter, and H. E. Umbarger (ed.), Escherichia coli and Salmonella: cellular and molecular biology, 2nd ed., vol. 1. ASM Press, Washington, D.C.

54. Jones, R. N., and A. L. Barry. 1987. Antimicrobial activity and spectrum of LY146032, a lipopeptide antibiotic, including susceptibility testing recommendations. Antimicrob. Agents Chemother. 31:625-629.

55. Jung, D., A. Rozek, M. Okon, and R. E. Hancock. 2004. Structural transitions as determinants of the action of the calcium-dependent antibiotic daptomycin. Chem. Biol. 11:949-957.

56. Kagan, R. M., and S. Clarke. 1994. Widespread occurrence of three sequence motifs in diverse S-adenosylmethionine-dependent methyltransferases suggests a common structure for these enzymes. Arch. Biochem. Biophys. 310:417-427.

57. Kahne, D., C. Leimkuhler, W. Lu, and C. Walsh. 2005. Glycopeptide and lipoglycopeptide antibiotics. Chem. Rev. 105:425-448.

58. Kasahara, T., and T. Kato. 2003. Nutritional biochemistry: A new redoxcofactor vitamin for mammals. Nature 422:832.

59. Kato, Y., and Y. Asano. 1997. 3-Methylaspartate ammonia-lyase as a marker enzyme of the mesaconate pathway for (S)-glutamate fermentation in Enterobacteriaceae. Arch. Microbiol. 168:457-463.

60. Keating, T. A., C. G. Marshall, C. T. Walsh, and A. E. Keating. 2002. The structure of $\mathrm{VibH}$ represents nonribosomal peptide synthetase condensation, cyclization and epimerization domains. Nat. Struct. Biol. 9:522-526.

61. Keller, S., T. Wage, K. Hohaus, M. Holzer, E. Eichhorn, and K. H. van Pee. 2000. Purification and partial characterization of tryptophan 7-halogenase (PrnA) from Pseudomonas fluorescens. Angew. Chem. Int. Ed. Engl. 39: 2300-2302.

62. Kessler, N., H. Schuhmann, S. Morneweg, U. Linne, and M. A. Marahiel. 2004. The linear pentadecapeptide gramicidin is assembled by four multimodular nonribosomal peptide synthetases that comprise 16 modules with 56 catalytic domains. J. Biol. Chem. 279:7413-7419.

63. Khosla, R., D. D. Verma, A. Kapur, R. V. Aruna, and N. Khanna. 1999 Streptogramins: a new class of antibiotics. Indian J. Med. Sci. 53:111-119.

64. Kim, H. B., C. P. Smith, J. Micklefield, and F. Mavituna, 2004 Metabolic flux analysis for calcium dependent antibiotic (CDA) production in Streptomyces coelicolor. Metab. Eng. 6:313-325.

65. Kohli, R. M., M. D. Burke, J. Tao, and C. T. Walsh. 2003. Chemoenzymatic route to macrocyclic hybrid peptide/polyketide-like molecules. J. Am. Chem. Soc. 125:7160-7161.

66. Kohli, R. M., J. Takagi, and C. T. Walsh. 2002. The thioesterase domain from a nonribosomal peptide synthetase as a cyclization catalyst for integrin binding peptides. Proc. Natl. Acad. Sci. USA 99:1247-1252.

67. Kohli, R. M., J. W. Trauger, D. Schwarzer, M. A. Marahiel, and C. T. Walsh. 2001. Generality of peptide cyclization catalyzed by isolated thioesterase domains of nonribosomal peptide synthetases. Biochemistry 40:70997108

68. Kohli, R. M., and C. T. Walsh. 2003. Enzymology of acyl chain macrocyclization in natural product biosynthesis. Chem. Commun. (Cambridge) 7:297-307.

69. Kohli, R. M., C. T. Walsh, and M. D. Burkart. 2002. Biomimetic synthesis and optimization of cyclic peptide antibiotics. Nature 418:658-661.

70. Konz, D., and M. A. Marahiel. 1999. How do peptide synthetases generate structural diversity? Chem. Biol. 6:R39-R48.

71. La Clair, J. J., T. L. Foley, T. R. Schegg, C. M. Regan, and M. D. Burkart. 
2004. Manipulation of carrier proteins in antibiotic biosynthesis. Chem. Biol. 11:195-201

72. Lambalot, R. H., A. M. Gehring, R. S. Flugel, P. Zuber, M. LaCelle, M. A Marahiel, R. Reid, C. Khosla, and C. T. Walsh. 1996. A new enzyme superfamily - the phosphopantetheinyl transferases. Chem. Biol. 3:923936.

73. Lin, H., D. A. Thayer, C. H. Wong, and C. T. Walsh. 2004. Macrolactamization of glycosylated peptide thioesters by the thioesterase domain of tyrocidine synthetase. Chem. Biol. 11:1635-1642.

74. Lin, H., and C. T. Walsh. 2004. A chemoenzymatic approach to glycopeptide antibiotics. J. Am. Chem. Soc. 126:13998-14003.

75. Lipmann, F. 1980. Bacterial production of antibiotic polypeptides by thiollinked synthesis on protein templates. Adv. Microb. Physiol. 21:227-266.

76. Lipmann, F., W. Gevers, H. Kleinkauf, and R. Roskoski, Jr. 1971. Polypeptide synthesis on protein templates: the enzymatic synthesis of gramicidin S and tyrocidine. Adv. Enzymol. Relat. Areas Mol. Biol. 35:1-34.

77. Liu, J., J. D. Farmer, Jr., W. S. Lane, J. Friedman, I. Weissman, and S. L. Schreiber. 1991. Calcineurin is a common target of cyclophilin-cyclosporin A and FKBP-FK506 complexes. Cell 66:807-815.

78. Liu, W., S. D. Christenson, S. Standage, and B. Shen. 2002. Biosynthesis of the enediyne antitumor antibiotic C-1027. Science 297:1170-1173.

79. Luo, L., R. M. Kohli, M. Onishi, U. Linne, M. A. Marahiel, and C. T. Walsh. 2002. Timing of epimerization and condensation reactions in nonribosomal peptide assembly lines: kinetic analysis of phenylalanine activating elongation modules of tyrocidine synthetase B. Biochemistry 41:91849196

80. Mahlert, C., S. A. Sieber, J. Grünewald, and M. A. Marahiel. 2005. Chemoenzymatic approach to enantiopure streptogramin B variants: characterization of stereoselective pristinamycin I cyclase from Streptomyces pristinaespiralis. J. Am. Chem. Soc. 127:9571-9580.

81. Marahiel, M. A., T. Stachelhaus, and H. D. Mootz. 1997. Modular peptide synthetases involved in nonribosomal peptide synthesis. Chem. Rev. 97: 2651-2674

82. Massey, V. 1994. Activation of molecular oxygen by flavins and flavoproteins. J. Biol. Chem. 269:22459-22462.

83. May, J. J., N. Kessler, M. A. Marahiel, and M. T. Stubbs. 2002. Crystal structure of DhbE, an archetype for aryl acid activating domains of modular nonribosomal peptide synthetases. Proc. Natl. Acad. Sci. USA 99:1212012125.

84. May, J. J., T. M. Wendrich, and M. A. Marahiel. 2001. The dhb operon of Bacillus subtilis encodes the biosynthetic template for the catecholic siderophore 2,3-dihydroxybenzoate-glycine-threonine trimeric ester bacillibactin. J. Biol. Chem. 276:7209-7217.

85. Mercer, A. C., J. J. La Clair, and M. D. Burkart. 2005. Fluorescent multiplex analysis of carrier protein post-translational modification. Chembiochem 6:1335-1337.

86. Miao, V., R. Brost, J. Chapple, K. She, M. F. Gal, and R. H. Baltz. 2005. The lipopeptide antibiotic A54145 biosynthetic gene cluster from Streptomyces fradiae. J. Ind. Microbiol. Biotechnol. 2005:1-12. [Online.]

87. Miao, V., M. F. Coeffet-Legal, P. Brian, R. Brost, J. Penn, A. Whiting, S. Martin, R. Ford, I. Parr, M. Bouchard, C. J. Silva, S. K. Wrigley, and R. H. Baltz. 2005. Daptomycin biosynthesis in Streptomyces roseosporus: cloning and analysis of the gene cluster and revision of peptide stereochemistry. Microbiology 151:1507-1523.

88. Micklefield, J. 2004. Daptomycin structure and mechanism of action revealed. Chem. Biol. 11:887-888.

89. Mootz, H. D., N. Kessler, U. Linne, K. Eppelmann, D. Schwarzer, and M. A. Marahiel. 2002. Decreasing the ring size of a cyclic nonribosomal peptide antibiotic by in-frame module deletion in the biosynthetic genes. J. Am. Chem. Soc. 124:10980-10981.

90. Mootz, H. D., D. Schwarzer, and M. A. Marahiel. 2000. Construction of hybrid peptide synthetases by module and domain fusions. Proc. Natl. Acad. Sci. USA 97:5848-5853.

91. Mootz, H. D., D. Schwarzer, and M. A. Marahiel. 2002. Ways of assembling complex natural products on modular nonribosomal peptide synthetases. Chembiochem 3:490-504.

92. Mukhtar, T. A., K. P. Koteva, and G. D. Wright. 2005. Chimeric streptogramin-tyrocidine antibiotics that overcome streptogramin resistance. Chem. Biol. 12:229-235.

93. Nowak-Thompson, B., N. Chaney, J. S. Wing, S. J. Gould, and J. E. Loper. 1999. Characterization of the pyoluteorin biosynthetic gene cluster of Pseudomonas fluorescens Pf-5. J. Bacteriol. 181:2166-2174.

94. Pelzer, S., R. Sussmuth, D. Heckmann, J. Recktenwald, P. Huber, G. Jung, and W. Wohlleben. 1999. Identification and analysis of the balhimycin biosynthetic gene cluster and its use for manipulating glycopeptide biosynthesis in Amycolatopsis mediterranei DSM5908. Antimicrob. Agents Chemother. 43:1565-1573.

95. Pfeifer, E., M. Pavela-Vrancic, H. von Dohren, and H. Kleinkauf. 1995 Characterization of tyrocidine synthetase 1 (TY1): requirement of posttranslational modification for peptide biosynthesis. Biochemistry 34:74507459

96. Price, J. C., E. W. Barr, B. Tirupati, J. M. Bollinger, Jr., and C. Krebs.
2003. The first direct characterization of a high-valent iron intermediate in the reaction of an alpha-ketoglutarate-dependent dioxygenase: a high-spin FeIV complex in taurine/alpha-ketoglutarate dioxygenase (TauD) from Escherichia coli. Biochemistry 42:7497-7508.

97. Puk, O., D. Bischoff, C. Kittel, S. Pelzer, S. Weist, E. Stegmann, R. D. Sussmuth, and W. Wohlleben. 2004. Biosynthesis of chloro-beta-hydroxytyrosine, a nonproteinogenic amino acid of the peptidic backbone of glycopeptide antibiotics. J. Bacteriol. 186:6093-6100.

98. Puk, O., P. Huber, D. Bischoff, J. Recktenwald, G. Jung, R. D. Sussmuth, K. H. van Pee, W. Wohlleben, and S. Pelzer. 2002. Glycopeptide biosynthesis in Amycolatopsis mediterranei DSM5908: function of a halogenase and a haloperoxidase/perhydrolase. Chem. Biol. 9:225-235.

99. Raja, A., J. LaBonte, J. Lebbos, and P. Kirkpatrick. 2003. Daptomycin. Nat. Rev. Drug Discov. 2:943-944.

100. Richardt, A., T. Kemme, S. Wagner, D. Schwarzer, M. A. Marahiel, and B. T. Hovemann. 2003. Ebony, a novel nonribosomal peptide synthetase for beta-alanine conjugation with biogenic amines in Drosophila. J. Biol. Chem. 278:41160-41166.

101. Rotondi, K. S., and L. M. Gierasch. 2005. A well-defined amphipathic conformation for the calcium-free cyclic lipopeptide antibiotic, daptomycin, in aqueous solution. Biopolymers 80:374-385.

102. Schauwecker, F., F. Pfennig, N. Grammel, and U. Keller. 2000. Construction and in vitro analysis of a new bi-modular polypeptide synthetase for synthesis of N-methylated acyl peptides. Chem. Biol. 7:287-297.

103. Schneider, A., T. Stachelhaus, and M. A. Marahiel. 1998. Targeted alteration of the substrate specificity of peptide synthetases by rational module swapping. Mol. Gen. Genet. 257:308-318.

104. Schneider, T. L., B. Shen, and C. T. Walsh. 2003. Oxidase domains in epothilone and bleomycin biosynthesis: thiazoline to thiazole oxidation during chain elongation. Biochemistry 42:9722-9730.

105. Schneider, T. L., and C. T. Walsh. 2004. Portability of oxidase domains in nonribosomal peptide synthetase modules. Biochemistry 43:15946-15955.

106. Schwarzer, D., R. Finking, and M. A. Marahiel. 2003. Nonribosomal peptides: from genes to products. Nat. Prod. Rep. 20:275-287.

107. Schwarzer, D., and M. A. Marahiel. 2001. Multimodular biocatalysts for natural product assembly. Naturwissenschaften 88:93-101.

108. Schwarzer, D., H. D. Mootz, U. Linne, and M. A. Marahiel. 2002. Regeneration of misprimed nonribosomal peptide synthetases by type II thioesterases. Proc. Natl. Acad. Sci. USA 99:14083-14088.

109. Sewald, N., and H. Jakubke. 2002. Peptides: chemistry and biology, p. 313. Wiley-VCH, Weinheim, Germany.

110. Sieber, S. A., and M. A. Marahiel. 2003. Learning from nature's drug factories: nonribosomal synthesis of macrocyclic peptides. J. Bacteriol. 185: 7036-7043.

111. Sieber, S. A., and M. A. Marahiel. 2005. Molecular mechanisms underlying nonribosomal peptide synthesis: approaches to new antibiotics. Chem. Rev. 105:715-738.

112. Sieber, S. A., J. Tao, C. T. Walsh, and M. A. Marahiel. 2004. Peptidyl thiophenols as substrates for nonribosomal peptide cyclases. Angew. Chem. Int. Ed. Engl. 43:493-498.

113. Sieber, S. A., C. T. Walsh, and M. A. Marahiel. 2003. Loading peptidylcoenzyme A onto peptidyl carrier proteins: a novel approach in characterizing macrocyclization by thioesterase domains. J. Am. Chem. Soc. 125: $10862-10866$.

114. Siedlecki, J., J. Hill, I. Parr, X. Yu, M. Morytko, Y. Zhang, J. Silverman, N. Controneo, V. Laganas, T. Li, J. Li, D. Keith, G. Shimer, and J. Finn. 2003 Array synthesis of novel lipodepsipeptide. Bioorg. Med. Chem. Lett. 13: 4245-4249.

115. Silakowski, B., H. U. Schairer, H. Ehret, B. Kunze, S. Weinig, G. Nordsiek, P. Brandt, H. Blocker, G. Hofle, S. Beyer, and R. Muller. 1999. New lessons for combinatorial biosynthesis from myxobacteria. The myxothiazol biosynthetic gene cluster of Stigmatella aurantiaca DW4/3-1. J. Biol. Chem. 274: 37391-37399.

116. Stachelhaus, T., A. Huser, and M. A. Marahiel. 1996. Biochemical characterization of peptidyl carrier protein (PCP), the thiolation domain of multifunctional peptide synthetases. Chem. Biol. 3:913-921.

117. Stachelhaus, T., H. D. Mootz, V. Bergendahl, and M. A. Marahiel. 1998. Peptide bond formation in nonribosomal peptide biosynthesis. Catalytic role of the condensation domain. J. Biol. Chem. 273:22773-22781.

118. Stachelhaus, T., H. D. Mootz, and M. A. Marahiel. 1999. The specificityconferring code of adenylation domains in nonribosomal peptide synthetases. Chem. Biol. 6:493-505.

119. Stachelhaus, T., A. Schneider, and M. A. Marahiel. 1995. Rational design of peptide antibiotics by targeted replacement of bacterial and fungal domains. Science 269:69-72.

120. Stachelhaus, T., and C. T. Walsh. 2000. Mutational analysis of the epimerization domain in the initiation module PheATE of gramicidin S synthetase. Biochemistry 39:5775-5787.

121. Stein, T., J. Vater, V. Kruft, A. Otto, B. Wittmann-Liebold, P. Franke, M. Panico, R. McDowell, and H. R. Morris. 1996. The multiple carrier model of nonribosomal peptide biosynthesis at modular multienzymatic templates. J. Biol. Chem. 271:15428-15435. 
122. Storm, D. R., and J. L. Strominger. 1974. Binding of bacitracin to cells and protoplasts of Micrococcus lysodeikticus. J. Biol. Chem. 249:1823-1827.

123. Storm, D. R., and J. L. Strominger. 1973. Complex formation between bacitracin peptides and isoprenyl pyrophosphates. The specificity of lipidpeptide interactions. J. Biol. Chem. 248:3940-3945.

124. Stryer, L. 1996. Biochemie. Spektrum Akademischer Verlag, Heidelberg, Germany.

125. Süssmuth, R., S. Pelzer, G. Nicholson, T. Walk, W. Wohlleben, and G. Jung. 1999. New advances in the biosynthesis of glycopeptide antibiotics of the vancomycin type from Amycolatopsis mediterranei. Angew. Chem. 38:1976-1979.

126. Tally, F. P., and M. F. DeBruin. 2000. Development of daptomycin for gram-positive infections. J. Antimicrob. Chemother. 46:523-526.

127. Trauger, J. W., R. M. Kohli, H. D. Mootz, M. A. Marahiel, and C. T. Walsh. 2000. Peptide cyclization catalysed by the thioesterase domain of tyrocidine synthetase. Nature 407:215-218.

128. Trauger, J. W., R. M. Kohli, and C. T. Walsh. 2001. Cyclization of backbone-substituted peptides catalyzed by the thioesterase domain from the tyrocidine nonribosomal peptide synthetase. Biochemistry 40:7092-7098.

129. Trivedi, O. A., P. Arora, V. Sridharan, R. Tickoo, D. Mohanty, and R. S. Gokhale. 2004. Enzymic activation and transfer of fatty acids as acyladenylates in mycobacteria. Nature 428:441-445.

130. Tseng, C. C., S. D. Bruner, R. M. Kohli, M. A. Marahiel, C. T. Walsh, and S. A. Sieber. 2002. Characterization of the surfactin synthetase C-terminal thioesterase domain as a cyclic depsipeptide synthase. Biochemistry 41: $13350-13359$.

131. Turner, N. J. 2004. Enzyme catalysed deracemisation and dynamic kinetic resolution reactions. Curr. Opin. Chem. Biol. 8:114-119.

132. Uguru, G. C., C. Milne, M. Borg, F. Flett, C. P. Smith, and J. Micklefield. 2004. Active-site modifications of adenylation domains lead to hydrolysis of upstream nonribosomal peptidyl thioester intermediates. J. Am. Chem. Soc. 126:5032-5033.

133. Vaillancourt, F. H., E. Yeh, D. A. Vosburg, S. E. O'Connor, and C. T. Walsh. 2005. Cryptic chlorination by a non-haem iron enzyme during cyclopropyl amino acid biosynthesis. Nature 436:1191-1194.

134. Vaillancourt, F. H., J. Yin, and C. T. Walsh. 2005. SyrB2 in syringomycin E biosynthesis is a nonheme FeII alpha-ketoglutarate- and O2-dependent halogenase. Proc. Natl. Acad. Sci. USA 102:10111-10116.

135. Velkov, T., and A. Lawen. 2003. Non-ribosomal peptide synthetases as technological platforms for the synthesis of highly modified peptide bioeffectors-cyclosporin synthetase as a complex example. Biotechnol. Annu. Rev. 9:151-197.

136. Vertesy, L., E. Ehlers, H. Kogler, M. Kurz, J. Meiwes, G. Seibert, M. Vogel, and P. Hammann. 2000. Friulimicins: novel lipopeptide antibiotics with peptidoglycan synthesis inhibiting activity from Actinoplanes friuliensis sp. nov. II. Isolation and structural characterization. J. Antibiot. (Tokyo) 53: 816-827.

137. Vivero-Pol, L., N. George, H. Krumm, K. Johnsson, and N. Johnsson. 2005. Multicolor imaging of cell surface proteins. J. Am. Chem. Soc. 127:12770 12771.

138. Walsh, C. 2000. Molecular mechanisms that confer antibacterial drug resistance. Nature 406:775-781.
139. Walsh, C. T. 2004. Polyketide and nonribosomal peptide antibiotics: modularity and versatility. Science 303:1805-1810.

140. Weber, G., and E. Leitner. 1994. Disruption of the cyclosporin synthetase gene of Tolypocladium niveum. Curr. Genet. 26:461-467.

141. Weber, T., R. Baumgartner, C. Renner, M. A. Marahiel, and T. A. Holak. 2000. Solution structure of PCP, a prototype for the peptidyl carrier domains of modular peptide synthetases. Structure Fold Des. 8:407-418.

142. Weist, S., B. Bister, O. Puk, D. Bischoff, S. Pelzer, G. J. Nicholson, W. Wohlleben, G. Jung, and R. D. Sussmuth. 2002. Fluorobalhimycin-a new chapter in glycopeptide antibiotic research. Angew. Chem. Int. Ed. Engl. 41:3383-3385.

143. Williams, D. H., M. Williamson, D. W. Butcher, and S. J. Hammond. 1983. Detailed binding sites of the antibiotics vancomycin and ristocetin A: determination of intermolecular distances in antibiotic/substrate complexes by use of the time-dependent nuclear Overhauser effect (NOE). J. Am. Chem. Soc. 105:1332-1339.

144. Wohlleben, W., and S. Pelzer. 2002. New compounds by combining "modern" genomics and "old-fashioned" mutasynthesis. Chem. Biol. 9:11631164.

145. Wyckoff, E. E., J. A. Stoebner, K. E. Reed, and S. M. Payne. 1997. Cloning of a Vibrio cholerae vibriobactin gene cluster: identification of genes required for early steps in siderophore biosynthesis. J. Bacteriol. 179:70557062.

146. Wynands, I., and K. H. van Pee. 2004. A novel halogenase gene from the pentachloropseudilin producer Actinoplanes sp. ATCC 33002 and detection of in vitro halogenase activity. FEMS Microbiol. Lett. 237:363-367.

147. Yeh, E., S. Garneau, and C. T. Walsh. 2005. Robust in vitro activity of RebF and $\mathrm{RebH}$, a two-component reductase/halogenase, generating 7-chlorotryptophan during rebeccamycin biosynthesis. Proc. Natl. Acad. Sci. USA 102:3960-3965.

148. Yeh, E., R. M. Kohli, S. D. Bruner, and C. T. Walsh. 2004. Type II thioesterase restores activity of a NRPS module stalled with an aminoacylS-enzyme that cannot be elongated. Chembiochem 5:1290-1293.

149. Yeh, E., H. Lin, S. L. Clugston, R. M. Kohli, and C. T. Walsh. 2004 Enhanced macrocyclizing activity of the thioesterase from tyrocidine synthetase in presence of nonionic detergent. Chem. Biol. 11:1573-1582.

150. Yin, J., A. J. Lin, P. D. Buckett, M. Wessling-Resnick, D. E. Golan, and C. T. Walsh. 2005. Single-cell FRET imaging of transferrin receptor trafficking dynamics by Sfp-catalyzed, site-specific protein labeling. Chem. Biol. 12:999-1006.

151. Yin, J., F. Liu, X. Li, and C. T. Walsh. 2004. Labeling proteins with small molecules by site-specific posttranslational modification. J. Am. Chem. Soc. 126:7754-7755.

152. Yin, J., F. Liu, M. Schinke, C. Daly, and C. T. Walsh. 2004. Phagemid encoded small molecules for high throughput screening of chemical libraries. J. Am. Chem. Soc. 126:13570-13571.

153. Zerbe, K., K. Woithe, D. B. Li, F. Vitali, L. Bigler, and J. A. Robinson. 2004. An oxidative phenol coupling reaction catalyzed by oxyB, a cytochrome P450 from the vancomycin-producing microorganism. Angew. Chem. Int. Ed. Engl. 43:6709-6713. 